\title{
IBEX OBSERVATIONS OF SECONDARY INTERSTELLAR HELIUM AND OXYGEN DISTRIBUTIONS
}

\author{
Jeewoo Park ${ }^{1}$, Harald Kucharek ${ }^{1}$, Eberhard Möbius ${ }^{1}$, André Galli $^{2}$, Marzena A. Kubiak ${ }^{3}$, \\ MaCIEJ BZOWSKI ${ }^{3}$, and David J. McComas ${ }^{4}$ \\ ${ }^{1}$ Space Science Center and Department of Physics, University of New Hampshire, 8 College Road, Durham, NH 03824, USA; jt129@wildcats.unh.edu \\ ${ }^{2}$ Physics Institute, University of Bern, Bern 3012, Switzerland \\ ${ }^{3}$ Space Research Centre of the Polish Academy of Science, Warsaw, Poland \\ ${ }^{4}$ Department of Astrophysical Sciences, Princeton University, Princeton, NJ 08544, USA \\ Received 2016 May 24; revised 2016 September 20; accepted 2016 October 8; published 2016 December 13
}

\begin{abstract}
In this study, we investigate the directional distributions of the secondary interstellar neutral (ISN) $\mathrm{He}$ and $\mathrm{O}$ populations at Earth's orbit. The secondary populations are created by charge exchange between ISN atoms and interstellar ions in the outer heliosheath. Using the IBEX-Lo He and O observations during the winter-spring seasons (early December to early June) in 2009-2011, we produced all-sky maps for He and O atoms with sputtering corrections. These sky maps include the directional distributions of the primary ISN gas and secondary populations. Our investigations reveal that the secondary $\mathrm{He}$ and $\mathrm{O}$ populations are observed in the ecliptic longitude range $160^{\circ}-210^{\circ}$. The peak longitudes of the secondary He and $\mathrm{O}$ appear to be $14^{\circ}-34^{\circ}$ and $38^{\circ}-43^{\circ}$ away from the peak longitude of the primary interstellar gas flow, respectively. These results indicate that the secondary populations have lower bulk speeds relative to the Sun and their flow directions deviate from the primary gas flow. These results may indicate that one side of the outer heliosheath is thicker than the other side relative to the flow direction of the primary interstellar gas flow.
\end{abstract}

Key words: ISM: atoms - ISM: general - ISM: structure - Sun: heliosphere

\section{INTRODUCTION}

The Interstellar Boundary EXplorer (IBEX), one of NASA's Small Explorer missions, was designed to discover the global interaction between the solar wind and the interstellar medium (McComas et al. 2009a). Since 2008 December, IBEX has been providing all-sky energetic neutral atom (ENA) images every six months by direct sampling of the local interstellar neutral (ISN) atoms. IBEX-Lo, one of two highly sensitive single-pixel cameras on $I B E X$, is optimized for the observation of neutral atoms in the energy range $0.01-2 \mathrm{keV}$ in eight partly overlapping, logarithmically spaced energy steps (Fuselier et al. 2009). Figure 1 shows the normalized transmission functions, which reflect the distributions of accepted energies relative to the central energies of the eight energy steps. The analytical expression of the transmission function is given by Schwadron et al. (2013).

In the IBEX-Lo energy range, we expect to observe three different populations of neutral atoms. First, heliospheric ENAs are clearly observed in the top four energy steps of IBEX-Lo (McComas et al. 2009b; Fuselier et al. 2012, 2014; Galli et al. 2014, 2016), roughly corresponding to the energy range $0.05-2 \mathrm{keV}$. The heliospheric ENAs are created by charge exchange between ISN atoms and solar wind ions in the inner heliosheath (Gruntman et al. 2001; Malama et al. 2006; Izmodenov et al. 2009; Chalov et al. 2010; Heerikhuisen et al. 2010). Second, $I B E X$-Lo is observing the primary ISN atoms, such as $\mathrm{H}, \mathrm{He}, \mathrm{O}$, and Ne. Through the IBEX-Lo observations, the ISN flow parameters (flow speed, direction, and temperature) have been investigated (Bzowski et al. 2012, 2014, 2015; Lee et al. 2012, 2015; Möbius et al. 2012, 2015a, 2015b; Katushkina et al. 2014; Leonard et al. 2015; McComas et al. 2015a, 2015b; Wood et al. 2015; etc.). For this study, we adopt a working value of the ISN gas flow speed to be $25.4 \mathrm{~km} \mathrm{~s}^{-1}$ (McComas et al. 2015b). Due to the solar gravitation, the flow speed becomes $\sim 49 \mathrm{~km} \mathrm{~s}^{-1}$ in the solar inertial frame at Earth's orbit. In the winter-spring season (early December to early June), the Earth moves into the gas flow. During this season, the apparent flow speed in the observer frame is $\sim 79 \mathrm{~km} \mathrm{~s}^{-1}$ and the corresponding bulk energies of the ISN $\mathrm{He}, \mathrm{O}$, and $\mathrm{Ne}$ atoms are $\sim 131 \mathrm{eV}, \sim 523 \mathrm{eV}$, and $\sim 653 \mathrm{eV}$, respectively, where we ignore the very small solar radiation pressure. For $\mathrm{H}$, for which the solar radiation pressure almost perfectly compensates for the solar gravity, the bulk energy is equal to $\sim 16 \mathrm{eV}$. Based on these energies, ISN $\mathrm{H}$ and $\mathrm{He}$ atoms have been observed in the lower energy steps of IBEX-Lo, corresponding to the energy range $15-110 \mathrm{eV}$ (Möbius et al. 2012; Saul et al. 2012; Schwadron et al. 2013). In addition, the ISN O and $\mathrm{Ne}$ gas is observed in the energy range $280-600 \mathrm{eV}$ (Möbius et al. 2009, 2012; Bochsler et al. 2012; Park et al. 2014, 2015). However, IBEX moves away from the ISN inflow in October, and then the flow speed in the observer frame is $\sim 19 \mathrm{~km} \mathrm{~s}^{-1}$. During the fall season, the energies (concerning $\mathrm{He}$ ) and intensities (concerning $\mathrm{O}$ ) are too low for a clear detection in the IBEX-Lo sensor (Galli et al. 2015). In Figure 1, we mark the expected energies for the ISN atoms and the heliospheric ENAs in the observer frame during the winterspring season.

Finally, the IBEX-Lo observations also show additional populations associated with neutral $\mathrm{He}$ and $\mathrm{O}$. Bzowski et al. (2012) hypothesized the secondary population of the ISN He atoms to describe the differences between simulations and observations. Kubiak et al. (2014) discovered what they termed the "Warm Breeze," which is slower and warmer than the primary ISN He population, and its flow direction differs by about $19^{\circ}$ from the ISN gas flow. Kubiak et al. (2016) revisited the inflow direction of the Warm Breeze and found that it is almost co-planar with the inflow directions of ISN $\mathrm{H}$ and $\mathrm{He}$ and the direction to the center of IBEX Ribbon. They concluded that the Warm Breeze is most likely the secondary population of ISN $\mathrm{He}$, created via charge exchange between $\mathrm{He}$ and $\mathrm{He}^{+}$ 

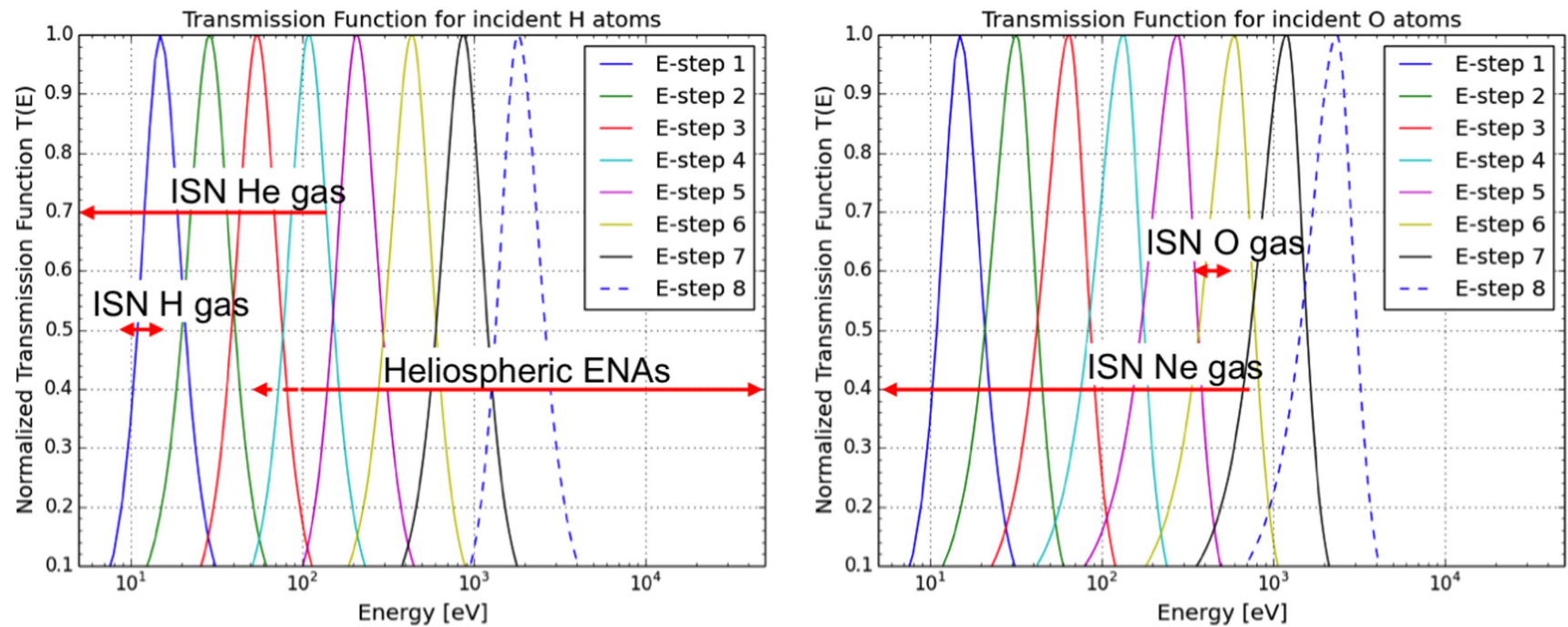

Figure 1. Transmission functions of the eight $I B E X$-Lo energy steps for " $\mathrm{H}^{--}$" events (left panel) and " $\mathrm{O}^{-}$" events (right panel). The normalized energy transmission function is taken from Schwadron et al. (2013). The energies differ for $\mathrm{H}$ and $\mathrm{O}$ because the energy loss on the conversion surface of the IBEX-Lo sensor depends on the atom species. The red arrows represent the possible energies of several populations of neutral atoms in the observer frame during the winter-spring season.

in the outer heliosheath. Park et al. (2015) studied the combined count-rate maps of heavy neutral atoms using three statistical analysis methods. They found an extended tail of the ISN O gas flow, centered around $190^{\circ}$ in ecliptic longitude and $+15^{\circ}$ in ecliptic latitude, or approximately $38^{\circ}$ from the ISN O and Ne gas flow peak. The most likely sources for these two populations are the secondary populations of ISN $\mathrm{He}$ and $\mathrm{O}$, which are created by charge exchange between ISN atoms (He and $\mathrm{H}$ ) and interstellar ions $\left(\mathrm{He}^{+}\right.$and $\left.\mathrm{O}^{+}\right)$in the outer heliosheath. The two-shock heliospheric model has been proposed to explain the existence of secondary ISN O and the O wall in front of the heliopause (Izmodenov et al. 1997, 1999, 2004). Therefore, instead of "Warm Breeze" and "extended tail," in this study, we call them the secondary ISN $\mathrm{He}$ and $\mathrm{O}$ populations, respectively. So far, these secondary $\mathrm{He}$ and $\mathrm{O}$ populations have been separately studied, and their directional distributions at Earth's orbit have not been compared in all-sky ENA images.

The IBEX-Lo sensor can distinguish species of the incoming neutral atoms through a time-of-flight (TOF) spectrometer after conversion to negative ions (Fuselier et al. 2009). This species identification is a very powerful capability of $I B E X$-Lo, but it is not unambiguous because of simultaneously generated sputtering products. On the conversion surface of IBEX-Lo, either the incoming neutral atoms are converted to negative ions, or they sputter negative ions. For $\mathrm{H}$ and $\mathrm{O}$ atoms, because most of the ions leaving the conversion surface are converted to negative ions, they are directly identified as converted $\mathrm{H}^{-}$and $\mathrm{O}^{-}$ions, respectively. However, because noble gas atoms, such as $\mathrm{He}$ and $\mathrm{Ne}$, do not produce stable negative ions, they are identified by the characteristic composition of sputtered $\mathrm{H}^{-}, \mathrm{C}^{-}$, and $\mathrm{O}^{-}$ ions. Conversely, $\mathrm{He}$ and $\mathrm{Ne}$ sputtering products can also masquerade as genuinely converted $\mathrm{H}^{-}$and $\mathrm{O}^{-}$. Therefore, a careful determination and treatment of the contribution of sputtering products are essential for the analysis of the IBEXLo observations.

In this paper, we examine the directional distributions of the secondary ISN $\mathrm{He}$ and $\mathrm{O}$ populations in the observer (or spacecraft) reference frame. The $I B E X$-Lo observations have provided the count-rate maps of the neutral $\mathrm{He}$ and $\mathrm{O}$ atoms. In these maps, we remove the apparent signals from sputtering of ENAs and ISN atoms. However, we do not apply a ComptonGetting correction, and all maps are shown in the observer reference frame. Here, we focus on the He maps in the lower four energy steps and the $\mathrm{O}$ maps in E-steps 5 and 6 . The overall structure of this paper is as follows. In Section 2, we briefly describe the IBEX-Lo sensor. In Section 3, we illustrate the data processing techniques that are used to produce the sky maps with the sputtering correction. In Section 4, we compare the directional distributions of the neutral $\mathrm{He}$ and $\mathrm{O}$ atoms in the sky maps. In Section 5, we discuss the possible flow peak longitudes of the secondary ISN populations in the outer heliosheath. Finally, Section 6 provides the conclusions from this investigation.

\section{INSTRUMENT}

The IBEX spacecraft is in a highly elliptical orbit around Earth with an apogee of $\sim 50$ Earth radii (McComas et al. 2009a). The orbit period initially was $\sim 7$ days and changed to $\sim 9$ days in 2011 June (McComas \& IBEX Science Team 2012). The IBEX's spin axis points toward the Sun. The two highly sensitive single-pixel cameras on IBEX look perpendicular to the spin axis and measure ENAs in the energy range of $10 \mathrm{eV}-6 \mathrm{keV}$. IBEX-Hi and -Lo have a $\sim 7^{\circ} \mathrm{FWHM}$ field of view and on each orbit scan one (before 2011 June) or two (after) different $360^{\circ} \times \sim 7^{\circ}$ swaths of the sky. Over six months, therefore, the two cameras scan the entire sky and provide all-sky maps in neutral atoms.

The IBEX-Lo sensor is composed of a collimator, a conversion surface, an electrostatic analyzer (ESA), and a TOF mass spectrometer. The collimator rejects incoming ions $(E<10 \mathrm{keV})$ and electrons $(E<600 \mathrm{eV})$. Neutral atoms pass the collimator and hit the conversion surface where a certain fraction of them is converted to negative ions. The conversion is rather efficient for $\mathrm{H}$ and $\mathrm{O}$ because these species form stable negative ions. However, it is inefficient for the noble gas atoms, such as $\mathrm{He}$ and $\mathrm{Ne}$, because they have no stable or metastable negative ions configuration. Instead, $\mathrm{He}$ and $\mathrm{Ne}$ atoms sputter negative ions from the conversion surface. Negative ions are accelerated and then passed through the toroidal ESA, which 
Table 1

Orbits and Dates of IBEX Observations with the Corresponding Map Numbers

\begin{tabular}{lcc}
\hline \hline Map \# & Orbits & Dates \\
\hline 1 & $13-33$ & 2009 Jan 09-2009 Jun 18 \\
3 & $57-80$ & 2009 Dec 10-2010 Jun 11 \\
5 & (exclude 62, 75, 79) & \\
& 2010 Dec 04-2011 Jan 18 \\
& & 2011 Feb 26-2011 Jun 05 \\
\hline
\end{tabular}

has eight logarithmically spaced energy steps with a width $\Delta E / E \approx 0.7$ (see Figure 1). The negative ions exiting the ESA are post-accelerated through a $16 \mathrm{kV}$ high voltage and enter the TOF mass spectrometer. The TOF spectrometer determines the ion masses. In 2012 June, we reduced the post-acceleration voltage to $7 \mathrm{kV}$, and since then the $I B E X$-Lo efficiency for $\mathrm{O}$ atoms has been too low for meaningful observations. Also, $I B E X$ was maneuvered into an essentially stable long-term orbit in 2011 June (McComas et al. 2011). Although IBEX-Lo is still observing ENAs, we only use the IBEX-Lo observations from the first three years (2009-2011) in this study. For more details concerning IBEX-Lo, we refer the reader to the instrument paper (Fuselier et al. 2009).

\section{METHODOLOGY}

In this section, we discuss the data selection criteria and the map generation techniques with the sputtering corrections. We use the IBEX-Lo observations during the winter-spring (early December to early June) seasons in 2009-2011.

\subsection{Data Selection}

To suppress noise and background we only use the triple coincidence (three valid TOF values) events. The triple coincidence events also satisfy the criterion: TOF0 + TOF3 $=$ TOF1 + TOF2. Three TOFs (TOF0, TOF1, and TOF2) are determined between two carbon foils and the micro-channel plate detector in the TOF mass spectrometer, and TOF3 is a delay line TOF measurement (Fuselier et al. 2009). In this analysis, we use the so-called "super good times" list to generate the all-sky ENA maps, which exclude neutral atoms generated by the Earth's magnetosphere, Moon, and interplanetary disturbances. The times when the instrument was observing these neutral atoms have been manually excluded. Also, the "super good times" list further excludes time intervals when the event rate exceeded three events per $6^{\circ}$ sector over a $96 \mathrm{~s}$ interval in the two highest energy steps (Estep 7 and 8) to remove various backgrounds from energetic ions or magnetospheric neutrals (Fuselier et al. 2012).

Table 1 provides the IBEX observation orbit numbers and the corresponding dates for three energy-resolved sets of six-month maps (map1, map3, and map5). The orbit numbers of these three maps are slightly different from the orbit numbers in the IBEX reference paper (McComas et al. 2014): map3 and map5 in our paper include the earlier orbits (orbit 57-58 for map3; orbit 104-106 for map5) because the secondary ISN populations are observed in early December. In map3, orbit 62 is excluded because of a reset of the onboard computer. Orbits 75 and 79 are also excluded because of contamination from the Earth's bow shock. Orbits 110-114 are discarded because the $I B E X$-Lo sensor was operated in a high angular resolution mode using only two energy steps (E-steps 2 and 6).

\subsection{Map Generation}

The sky maps obtained with IBEX-Lo include both converted and sputtered negative ions. These two components cannot be distinguished by the TOF mass spectrometer. However, we can identify these two populations in the sky map because of the different energy distributions of the converted and sputtered ions. The converted ion distribution is narrow and shifted slightly to lower energy than the incoming energy. However, the sputtered negative ions produce a broad energy distribution ranging from just below the incident energy to very low energies because the sputtered ions are released at the conversion surface with a widely varying fraction of the incoming atom energy (Möbius et al. 2012). For instance, for incoming $\mathrm{H}$ and $\mathrm{O}$ atoms, most of the ions leaving the conversion surface are converted $\mathrm{H}^{-}$and $\mathrm{O}^{-}$ions, respectively. The converted ions have an energy distribution shifted to lower energy (Möbius et al. 2012), which is approximately $15 \%$ lower than the incoming energy due to energy loss on the conversion surface (Fuselier et al. 2009). However, few of the $\mathrm{H}$ and $\mathrm{O}$ atoms are sputtered $\mathrm{H}^{-}, \mathrm{C}^{-}$, and $\mathrm{O}^{-}$ions, which have broad energy distributions lower than the incoming energy. On the other hand, the noble gas atoms ( $\mathrm{He}$ and $\mathrm{Ne}$ ) sputter $\mathrm{H}^{-}$, $\mathrm{C}^{-}$, and $\mathrm{O}^{-}$from the conversion surface, and these ions are observed in energy steps lower than the energy of the incoming noble gas atoms. Wurz et al. (2008) note that a few of $1500 \mathrm{eV}$ $\mathrm{He}$ atoms can be converted to negative $\mathrm{He}^{-}$ions in a metastable state, but this fraction decreases substantially with lower energies, including the He ISN flow energy. Therefore, we disregard the contribution from converted $\mathrm{He}^{-}$ions.

In our investigation, both converted and sputtered $\mathrm{H}^{-}$ negative ions are identified as " $\mathrm{H}^{-}$" events, and the $\mathrm{C}^{-}$and $\mathrm{O}^{-}$ negative ions are classified as " $\mathrm{O}^{-}$" events. Because of their closeness in mass, the $\mathrm{C}^{-}$and $\mathrm{O}^{-}$TOF distributions partially overlap in a specific TOF range (e.g., $50 \mathrm{~ns}<\mathrm{TOF} 2<100 \mathrm{~ns}$ in IBEX-Lo; Park et al. 2014), and they are not clearly separable. In addition, $\mathrm{C}^{-}$ions registered in the sensor are solely sputter products of elements such as $\mathrm{H}, \mathrm{He}, \mathrm{O}$, and $\mathrm{Ne}$. We do not expect to observe interstellar $\mathrm{C}$ atoms because of the high ionization fraction $\left(X_{\mathrm{C}}=0.975\right)$ of carbon in the Local Interstellar Cloud (Slavin \& Frisch 2008). To simplify our analysis, we classify $\mathrm{C}^{-}$and $\mathrm{O}^{-}$as " $\mathrm{O}^{-}$" events.

In the IBEX-Lo energy range, we expect to observe the ISN gas atoms $(\mathrm{H}, \mathrm{He}, \mathrm{O}$, and $\mathrm{Ne})$, the secondary ISN He and $\mathrm{O}$ populations, and the heliospheric ENAs (mostly $\mathrm{H}$ ). Based on the combined energy distribution of these populations, the observed $\mathrm{H}^{-}$rate map in E-step $i\left(C_{i}^{\mathrm{H}^{-}}\right)$can be written as

$$
\begin{aligned}
C_{i}^{\mathrm{H}^{-}}= & G_{i i}^{\mathrm{H}^{-} \mathrm{H}} E_{i} J_{i}^{\mathrm{H}}+\sum_{k>i} G_{i k}^{\mathrm{H}^{-} \mathrm{H}} E_{i} J_{k}^{\mathrm{H}}+\sum_{k \geqslant i} G_{i k}^{\mathrm{H}^{-} \mathrm{He}} E_{i} J_{k}^{\mathrm{He}} \\
& +\sum_{k \geqslant i} G_{i k}^{\mathrm{H}^{-} \mathrm{O}} E_{i} J_{k}^{\mathrm{O}}+\sum_{k \geqslant i} G_{i k}^{\mathrm{H}^{-} \mathrm{Ne}} E_{i} J_{k}^{\mathrm{Ne}},
\end{aligned}
$$

where $G_{i k}^{\mathrm{XS}}$ is the geometric factor (in units of $\mathrm{cm}^{2} \mathrm{sr} \mathrm{keV} \mathrm{keV}{ }^{-1}$ ) for the combination of an incoming atom " $\mathrm{S}$ " and an observed negative ion "X" (see the Appendices). $E_{i}$ is the center energy of E-step $i$. The index $i$ represents the E-step of the IBEX-Lo ESA $(i=1-8)$, and the index $k$ indicates the incident energy step. Even though the incoming atom energy distributions are continuous, we use discrete steps $k$ to represent the incoming energy spectra. The index $k$ can be $1-8$ and indicates the center energy of the corresponding ESA energy step (i.e., $k$ $=1-8$ and $E_{k}=E_{i}$ for $\left.i=k\right) . J_{i}^{\mathrm{S}}\left(J_{k}^{\mathrm{S}}\right)$ is a differential flux (in 
units of $\mathrm{cm}^{-2} \mathrm{~s}^{-1} \mathrm{sr}^{-1} \mathrm{keV}^{-1}$ ) of the incoming atom " $\mathrm{S}$," which has an energy corresponding to $E_{i}\left(E_{k}\right)$. On the right-hand side of Equation (1), the first term represents the count rate of the converted $\mathrm{H}^{-}$ions due to the incoming $\mathrm{H}$ atoms that have the same energy as the ESA E-step $i$. The second term is the count rate of the sputtered $\mathrm{H}^{-}$due to the $\mathrm{H}$ atoms that have higher energies than those in E-step $i(k>i)$. The other terms indicate the count rates of the sputtered $\mathrm{H}^{-}$ions due to $\mathrm{He}, \mathrm{O}$, and $\mathrm{Ne}$ atoms, respectively. Here, the incoming neutral atoms have energies higher than and equal to one corresponding to ESA E-step $i(k \geqslant i)$.

Similarly, the observed $\mathrm{O}^{-}$rate map in E-step $i\left(C_{i}^{\mathrm{O}^{-}}\right)$can also be written as

$$
\begin{aligned}
C_{i}^{\mathrm{O}^{-}}= & G_{i i}^{\mathrm{O}^{-} \mathrm{O}} E_{i} J_{i}^{\mathrm{O}}+\sum_{k>i} G_{i k}^{\mathrm{O}^{-} \mathrm{O}} E_{i} J_{k}^{\mathrm{O}}+\sum_{k \geqslant i} G_{i k}^{\mathrm{O}^{-} \mathrm{H}} E_{i} J_{k}^{\mathrm{H}} \\
& +\sum_{k \geqslant i} G_{i k}^{\mathrm{O}^{-} \mathrm{He}} E_{i} J_{k}^{\mathrm{He}}+\sum_{k \geqslant i} G_{i k}^{\mathrm{O}^{-} \mathrm{Ne}} E_{i} J_{k}^{\mathrm{Ne}}
\end{aligned}
$$

where $E_{i}$ is a center energy of E-step $i$ for $\mathrm{O}^{-}$ions in the ESA (Figure 1). On the right-hand side of Equation (2), the first term indicates the count rate of converted $\mathrm{O}^{-}$ions due to incoming $\mathrm{O}$ atoms that have the same energy as E-step $i$. The second term is the count rate of sputtered $\mathrm{O}^{-}$due to the $\mathrm{O}$ atoms that have higher energies than E-step $i$. The other terms represent the count rates of sputtered $\mathrm{O}^{-}$ions due to the $\mathrm{H}, \mathrm{Ne}$, and $\mathrm{Ne}$ atoms that have energies higher than and equal to E-step $i$.

In Equations (1) and (2), there are four terms representing the sputter contributions. In principle, ISN $\mathrm{Ne}$ atoms can be observed as " $\mathrm{O}^{-}$" events in E-steps 5-6 in accordance with the ISN Ne bulk energy. However, it is impossible to unambiguously distinguish the " $\mathrm{O}^{-}$" events caused by ISN $\mathrm{Ne}$ atoms from genuine " $\mathrm{O}^{-}$" events. Only the $\mathrm{Ne} / \mathrm{O}$ ratio in the primary ISN flow has been studied by Bochsler et al. (2012) and Park et al. (2014). In addition, because of much lower charge exchange cross-sections, the contribution of $\mathrm{Ne}$ is expected to be less important for the secondary component. Because we focus on the secondary ISN component, we do not consider the contribution of the ISN Ne atoms in this study. Therefore, the last terms in Equations (1) and (2) are neglected in the following equations.

In this study, the IBEX-Lo observations are shown in Mollweide projections in ecliptic coordinates $\lambda$ (longitude) and $\beta$ (latitude). The directions toward the nose $\left((\lambda, \beta)=\left(255^{\circ} .7\right.\right.$, $5.1))$, tail, and the two Voyager spacecraft are marked with filled red circles. The sky maps represent the count rates on a logarithmic scale in the spacecraft frame. Figure 2 shows the combined $\mathrm{H}^{-}$count-rate maps (left-hand side of Equation (1)) in the eight energy steps, which were created by combining the observed $\mathrm{H}^{-}$count-rate maps measured in 2009-2011 winterspring seasons (map1 + map3 + map5). Here we use the same combining technique as in Park et al. (2015). In the $\mathrm{H}^{-}$countrate maps for E-steps $1-4$, the strongest $\mathrm{H}^{-}$signal (red pixels) represents the ISN He gas flow. Heliospheric H ENAs are shown as light blue pixels in the $\mathrm{H}^{-}$maps in E-steps 5-8, with the ENA Ribbon as the most striking feature at these higher energies (McComas et al. 2014; Schwadron et al. 2014). Figure 3 illustrates the combined $\mathrm{O}^{-}$count-rate maps in the eight energy steps that were created by combining the observed $\mathrm{O}^{-}$count-rate maps (map $1+\operatorname{map} 3+$ map5). The $\mathrm{O}^{-}$signal in the $\mathrm{O}^{-}$maps in E-step $1-4$ indicates sputtered $\mathrm{O}^{-}$ions due to the ISN He flow. The ISN O and Ne gas flow is revealed by green pixels in the $\mathrm{O}^{-}$maps in E-steps 5-6.

\subsection{He All-sky Maps}

In the observed $\mathrm{H}^{-}$count-rate maps for the energy range 15-110 eV (E-steps 1-4), we expect to observe three populations: ISN He atoms, ISN $\mathrm{H}$ atoms, and heliospheric $\mathrm{H}$ atoms. During the winter-spring season, IBEX moves into the ISN gas flow and the bulk energy of the ISN He atoms is $\sim 131 \mathrm{eV}$ in the observer frame (corresponding to E-step 4). We expect to observe the ISN He atoms in the lower four energy steps $(15-110 \mathrm{eV})$ because $\mathrm{He}$ atoms sputter negative $\mathrm{H}^{-}$and $\mathrm{O}^{-}$ ions on the conversion surface, which have a broad energy distribution below the bulk energy. The bulk energy of the ISN $\mathrm{H}$ atoms is $\sim 16 \mathrm{eV}$, and the incoming $\mathrm{H}$ atoms are converted into the negative $\mathrm{H}^{-}$ions. Hence, we expect to observe these ISN $\mathrm{H}$ atoms in the two lowest energy steps (E-steps 1 and 2). Since the thermal speed of hydrogen is the largest among the four species due to its low atomic mass and, furthermore, the bulk speed of ISN $\mathrm{H}$ at $1 \mathrm{au}$ is twofold lower than the bulk speed of $\mathrm{He}, \mathrm{O}$, and $\mathrm{Ne}$ due to the action of radiation pressure on $\mathrm{H}$ but not on $\mathrm{He}$ and $\mathrm{O} \& \mathrm{Ne}$, the relative width of the ISN $\mathrm{H}$ distribution is much larger than the relative widths of the distributions of $\mathrm{He}$ and $\mathrm{O} \& \mathrm{Ne}$. Heliospheric $\mathrm{H}$ atoms contribute to both converted and sputtered $\mathrm{H}^{-}$, depending on their energies. In the lower four energy steps, the sputter contribution due to $\mathrm{O}$ and $\mathrm{Ne}$ is negligible due to their substantially lower abundance and higher bulk energy compared to $\mathrm{H}$ and $\mathrm{He}$. The detailed description is in the following paragraph. Therefore, the map of observed $\mathrm{H}^{-}$count rate in E-step $i$ includes converted $\mathrm{H}^{-}$ions due to the ISN and heliospheric $\mathrm{H}$ atoms with the same energy as E-step $i$, sputtered $\mathrm{H}^{-}$ions due to heliospheric $\mathrm{H}$ atoms at higher energies than E-step $i(k>i)$, and sputtered $\mathrm{H}^{-}$ions due to the ISN He atoms.

Now, we rewrite Equation (1) regarding the count rate through the typical definition of a differential flux $J_{k}^{\mathrm{S}}=C_{i}^{\mathrm{S}} /\left(G_{i k}^{\mathrm{XS}} E_{i}\right)$ (see Equation (18) in Appendix A). We also define a sputtering-correction factor $\alpha_{i k}^{\mathrm{XS}}$ to represent contributions of sputtered ions, which is discussed in the Appendix $\mathrm{A}$ in detail. For the observed $\mathrm{H}^{-}$count-rate maps in E-steps 1-4, Equation (1) becomes

$$
C_{i}^{\mathrm{H}^{-}}=C_{i}^{\mathrm{H}}+C_{i}^{\mathrm{He}}+\sum_{k>i}\left(\alpha_{i k}^{\mathrm{H}^{-} \mathrm{H}} C_{k}^{\mathrm{H}}\right)+\sum_{k \geqslant i}\left(\alpha_{i k}^{\mathrm{H}^{-} \mathrm{O}} C_{k}^{\mathrm{O}}\right) .
$$

On the right-hand side of Equation (3), the first term $C_{i}^{\mathrm{H}}$ is a count rate of $\mathrm{H}$ atoms in E-step $i$, which includes both the ISN $\mathrm{H}$ atoms and heliospheric $\mathrm{H}$ ENAs. The second term $C_{i}^{\mathrm{He}}$ is the count rate of He atoms, which is specified as

$$
C_{i}^{\mathrm{He}} \equiv G_{i, k=4}^{\mathrm{H}^{-} \mathrm{He}} E_{i} J_{k=4}^{\mathrm{He}},
$$

where $J_{k=4}^{\mathrm{He}}$ is the differential flux of the ISN He gas, and $G_{i, k=4}^{\mathrm{H}-\mathrm{He}}$ is the geometric factor for the sputtered $\mathrm{H}^{-}$ions due to the ISN He atoms.

On the right-hand side of Equation (3), the two terms in the summation are the sputtered contributions due to $\mathrm{H}$ and $\mathrm{O}$ atoms at higher energies, respectively. We ignore these two terms because the sputtering contributions due to $\mathrm{H}$ and $\mathrm{O}$ atoms are significantly smaller than the measured count rates of $\mathrm{H}^{-}$ions in E-steps 1-4. For instance, as shown in Figure 10(A), the sputtering-correction factor at E-step 4 due 

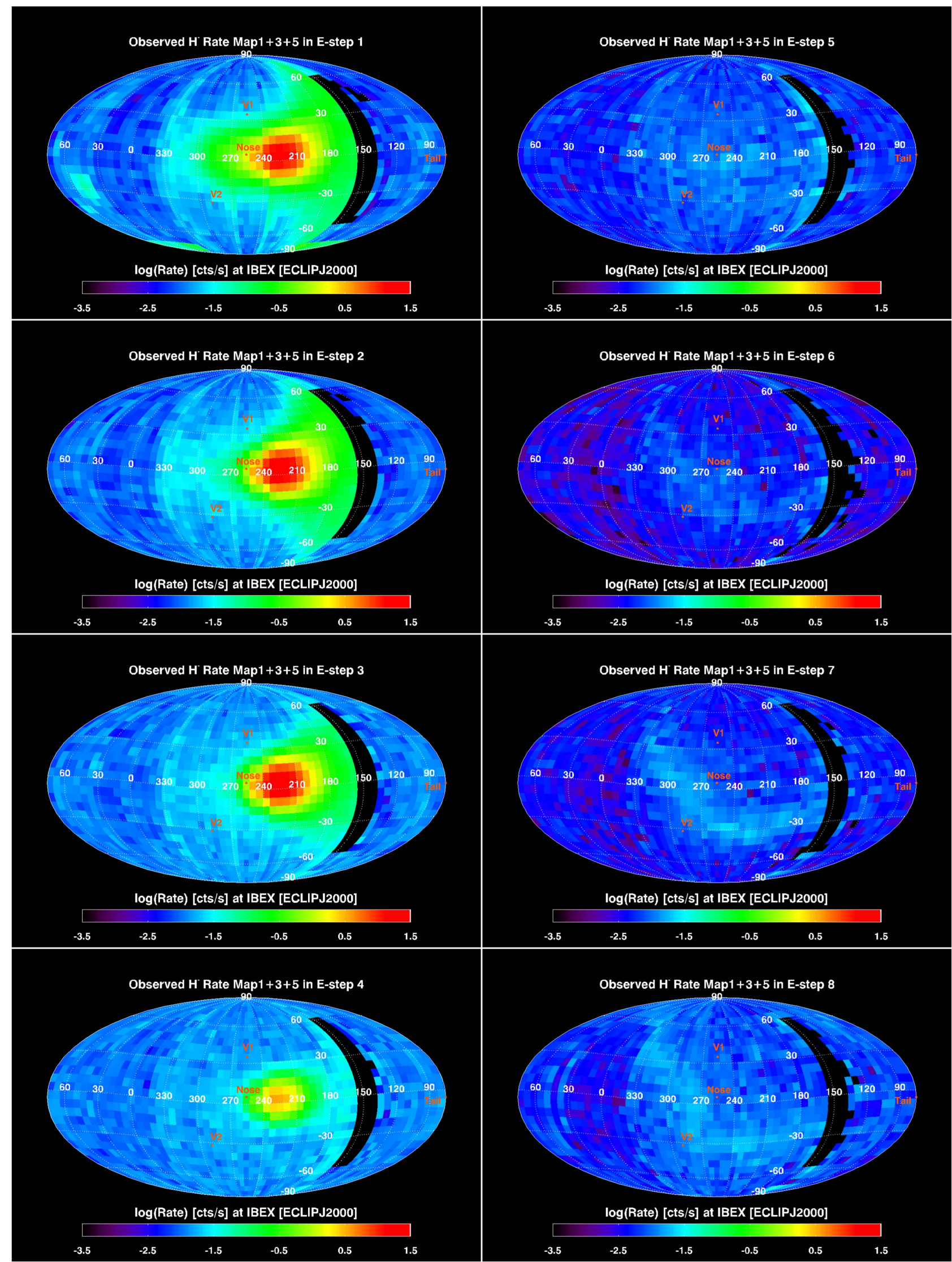

Figure 2. Combined $\mathrm{H}^{-}$count-rate maps for three consecutive winter-spring seasons from 2009 to 2011 in eight energy steps. These sky maps show the count-rate $\left(\mathrm{s}^{-1}\right)$ of $\mathrm{H}^{-}$ions in the reference frame of the IBEX spacecraft in Earth's orbit in a Mollweide projection. All panels use the same logarithmic color bar scaling from -3.5 to 1.5. The directions to the nose, tail, and the two Voyager spacecraft are marked with red circles. The black strips are discarded because of magnetospheric contamination. 


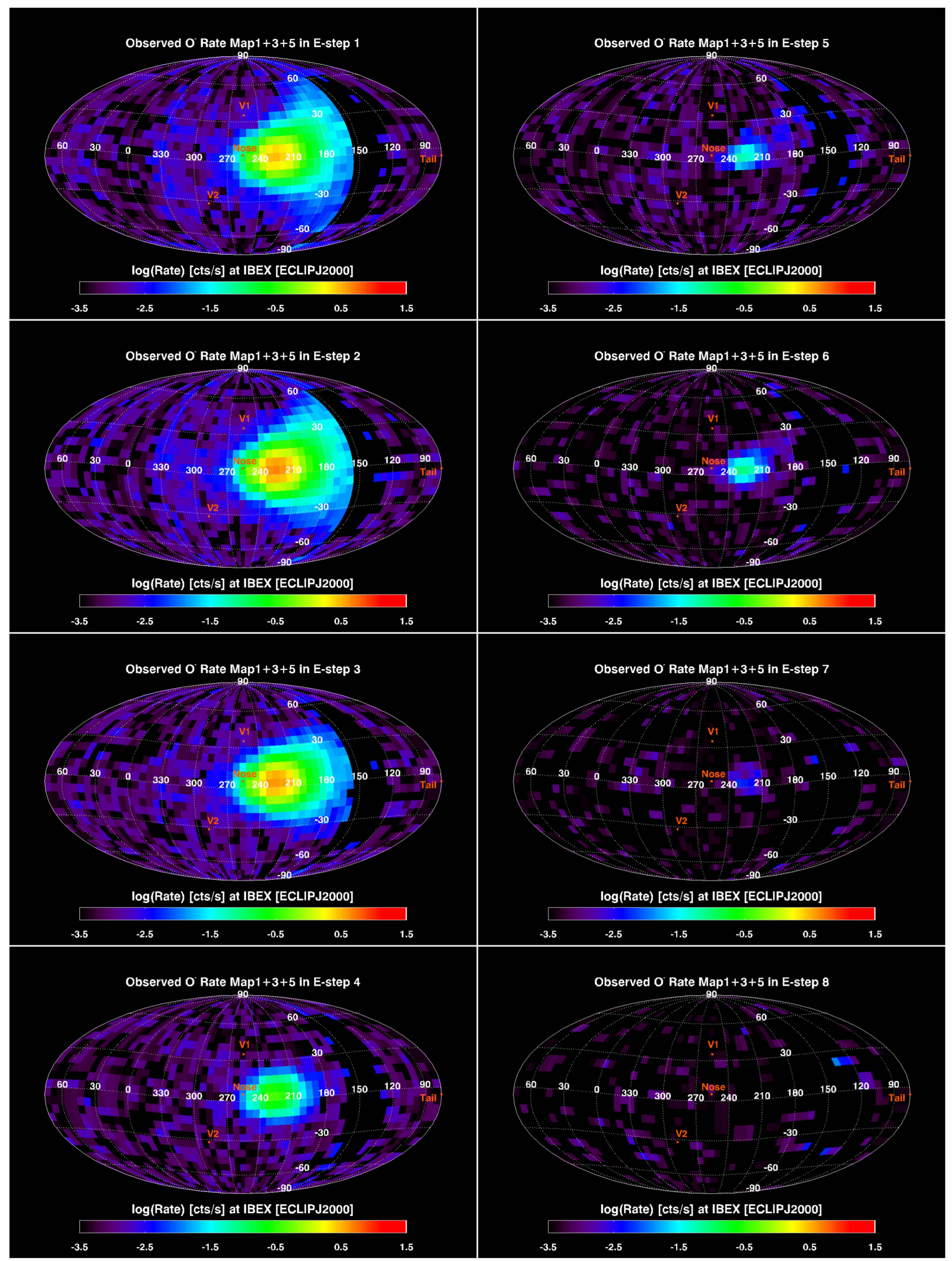

Figure 3. Observed $\mathrm{O}^{-}$count-rate maps for three consecutive winter-spring seasons from 2009 to 2011 in eight energy steps. The map projection style and legends are the same as in Figure 2. 
to $209 \mathrm{eV} \mathrm{H}$ atoms $\left(\alpha_{i=4, k=5}^{\mathrm{H}^{-} \mathrm{H}}\right)$ is $\sim 0.18$, and the other sputtering-correction factors due to $\mathrm{H}$ atoms at higher energies (i.e., $k>5$ ) are less than 0.02. The maximum count rate of measured $\mathrm{H}^{-}$ions in the $\mathrm{H}^{-}$all-sky map in E-step 5 is $0.034 \mathrm{~s}^{-1}$. Thus, the sputter contribution due to $\mathrm{H}$ atoms is approximately $6.12 \times 10^{-3} \mathrm{~s}^{-1}$. Galli et al. (2014, 2015) studied the spatial and temporal distribution of $\mathrm{H}$ ENAs from the heliosheath through IBEX-Lo observations. They inferred that the background count rates in the $\mathrm{H}^{-}$all-sky maps are $0.008-0.012 \mathrm{~s}^{-1}$, which is comparable to or greater than the sputter contribution due to $\mathrm{H}$ atoms at higher energies. Similarly, the sputter contributions due to $\mathrm{O}$ atoms at higher energies are also relatively small. In the observed $\mathrm{O}^{-}$count-rate maps in E-steps 5-8, the maximum count rate of $\mathrm{O}^{-}$is below $0.09 \mathrm{~s}^{-1}$. As illustrated in Figure 10(B), the sputteringcorrection factors $\alpha_{i k}^{\mathrm{H}^{-} \mathrm{O}}$ are below 0.05 . Therefore, the sputter contribution due to $\mathrm{O}$ atoms is on the order of $10^{-3} \mathrm{~s}^{-1}$, which is comparable to or less than the background count rates in the $\mathrm{H}^{-}$all-sky maps. Therefore, it is reasonable to ignore the sputtering contributions due to $\mathrm{H}$ and $\mathrm{O}$ atoms at higher energies in Equation (3). Consequently, the He count-rate map in E-step $i$ is estimated as

$$
C_{i}^{\mathrm{He}}=C_{i}^{\mathrm{H}^{-}}-G_{i i}^{\mathrm{H}^{-} \mathrm{H}} E_{i} J_{i}^{\mathrm{H}}
$$

and its variance is

$$
\sigma_{\mathrm{He}, i}^{2}=\sigma_{C^{\mathrm{H}^{-}, i}}^{2}+\left(G_{i i}^{\mathrm{H}^{-} \mathrm{H}} E_{i}\right)^{2} \cdot \sigma_{J^{\mathrm{H}}, i}^{2} .
$$

To estimate the He count rates, we need to know the H fluxes in E-step $i\left(J_{i}^{\mathrm{H}}\right)$, which are obtained from the $\mathrm{H}^{-}$count-rate maps in the higher energy steps.

For the higher energy steps (E-steps 5-8), the ISN He atoms cannot be observed due to their relatively low bulk energy. Unlike the lower energy steps, we need to consider the sputter contribution due to $\mathrm{H}$ atoms with energies higher than the energy step under consideration. However, the sputter contribution due to $\mathrm{O}$ atoms is still negligible. The reason is the low $\mathrm{O}$ abundance relative to $\mathrm{H}$. Therefore, Equation (1) is simplified as

$$
C_{i}^{\mathrm{H}}=C_{i}^{\mathrm{H}^{-}}-\sum_{k>i} \alpha_{i k}^{\mathrm{H}^{-} \mathrm{H}} C_{k}^{\mathrm{H}}
$$

where $\alpha_{i k}^{\mathrm{H}^{-} \mathrm{H}}$ is the sputtering-correction factor. The first term on the right-hand side is the observed $\mathrm{H}^{-}$count rate, and the second term illustrates the sputter contribution due to higher energy $\mathrm{H}$ atoms. We assume that there is no sputter contamination in the observed $\mathrm{H}^{-}$count rate in E-step 8, i.e., $C_{8}^{\mathrm{H}^{-}}=C_{8}^{\mathrm{H}}$. Then we produce the $\mathrm{H}$ count-rate maps in the lower energy steps sequentially through Equation (7) with the correction factors. The $\mathrm{H}$ flux map in E-step $i$ is estimated through the flux definition, $J_{i}^{\mathrm{H}}=C_{i}^{\mathrm{H}} /\left(G_{i i}^{\mathrm{H}^{-} \mathrm{H}} E_{i}\right)$, and its uncertainty is calculated as $\sigma_{J^{\mathrm{H}}, i}^{2}=\left(\sigma_{C^{\mathrm{H}}, i} /\left(G_{i i}^{\mathrm{H}^{-} \mathrm{H}} E_{i}\right)\right)^{2}$. Through this process, we estimate the $\mathrm{H}$ flux maps in E-steps 5-8, whose $\mathrm{H}$ fluxes represent the flux of the heliospheric ENAs.

We cannot use Equation (7) to estimate the H flux maps in E-steps 1-4 because ISN H and He gas atoms are also observed in these energy steps. Instead, we extend the heliospheric ENA spectrum as a power law to estimate the $\mathrm{H}$ fluxes in the lower energy steps. We assume that the relation between the $\mathrm{H}$ flux and energy in the log-log scale is written as

$$
\log J(E)=a_{0}+a_{1} \log E \text {. }
$$

We determined the parameter $a_{0}$ and $a_{1}$ in Equation (8) by using the method of least squares with the paired data $\left(\log E_{i}, \log J_{i}\right)$. The error of $\log J$ is $\sigma_{\log J}=\sigma_{J} /(J \cdot \ln 10)$. We performed a power-law fit to each pixel of the estimated $\mathrm{H}$ flux maps in E-steps 5-8. In other words, we did fit a power law to the differential H ENA fluxes $J$ of the four highest energy steps. The global average value of the estimated exponent $a_{1}$ is $-1.364 \pm 0.346$. For the lower energy steps, therefore, the $\mathrm{H}$ flux map is estimated by

$$
J_{i}^{\mathrm{H}}\left(E_{i}\right)=10^{a_{0}} E_{i}^{a_{1}}
$$

and its variance is

$$
\sigma_{J, i}^{2}=\left(J_{i}^{\mathrm{H}}\right)^{2} \cdot\left[(\ln 10)^{2} \sigma_{a_{0}}^{2}+\left(\ln E_{i}\right)^{2} \sigma_{a_{1}}^{2}\right]
$$

We insert these estimated $\mathrm{H}$ fluxes into Equation (5) to produce the He count-rate maps in E-steps 1-4. Figure 4 shows the sputtering-corrected He count-rate maps in these lower energy steps. In this step, we assume that the heliospheric ENAs follow a uniform power law from solar wind energies down to the lowest energy step of IBEX-Lo. Galli et al. (2016) found that the energy spectrum of heliospheric ENAs rolls over at $0.1 \mathrm{keV}$ and the energy spectra at several directions can be described by a power law with slope $-1.2 \pm 0.1$ between 0.1 and $1.8 \mathrm{keV}$. This simplifying assumption could lead us to overestimate the $\mathrm{H}$ fluxes in E-steps $1-3$, but the average slope from the $\mathrm{H}$ flux maps in E-steps $5-8$ is close to the slope inferred by Galli et al. (2016). Therefore, the overestimated $\mathrm{H}$ fluxes in lower energy steps do not lead to a significant effect on the directional distribution of $\mathrm{He}$ atoms in the all-sky $\mathrm{He}$ maps. In addition, this procedure provides the estimated fluxes of heliospheric $\mathrm{H}$ atoms in the lower energy steps, but it cannot take into account the fluxes of ISN $\mathrm{H}$ atoms. Therefore, the contribution due to the ISN $\mathrm{H}$ atoms remains in our sputtering correction.

\subsection{All-sky O Maps}

In the observed $\mathrm{O}^{-}$rate maps, unlike the observed $\mathrm{H}^{-}$rate maps, we expect to observe the ISN $\mathrm{O}$ and $\mathrm{Ne}$ gas atoms and the apparent $\mathrm{O}^{-}$signal from sputtering of ISN He atoms. There is no known source of heavy heliospheric ENAs. Due to the high masses of the ISN O and $\mathrm{Ne}$ atoms, the ISN O and $\mathrm{Ne}$ gas atoms have energies corresponding to E-steps 5 and 6. The ISN $\mathrm{O}$ and $\mathrm{Ne}$ gas is also observed in the map at E-step 7 because of the broad energy pass-band of the ESA $(\Delta E / E=0.7)$. The $\mathrm{O}$ maps in E-steps 1-4 show an apparent sputtered $\mathrm{O}^{-}$signal from sputtering of ISN He atoms (Figure 3). A fraction of the heliospheric $\mathrm{H}$ atoms can also produce sputtered $\mathrm{O}^{-}$ions, which are apparently seen in the observed $\mathrm{O}^{-}$count-rate maps. However, because the sputtering-correction factors for $\mathrm{O}^{-}$ due to the higher energy $\mathrm{H}$ atoms are less than 0.004 (see Figure 10(D)), we ignore this sputter contribution in the $\mathrm{O}^{-}$count-rate maps. Therefore, based on Equation (2), the $\mathrm{O}$ count-rate map in E-step $i$ is

$$
C_{i}^{\mathrm{O}}=C_{i}^{\mathrm{O}^{-}}-\sum_{k>i} \alpha_{i k}^{\mathrm{O}^{-} \mathrm{O}} C_{k}^{\mathrm{O}}
$$




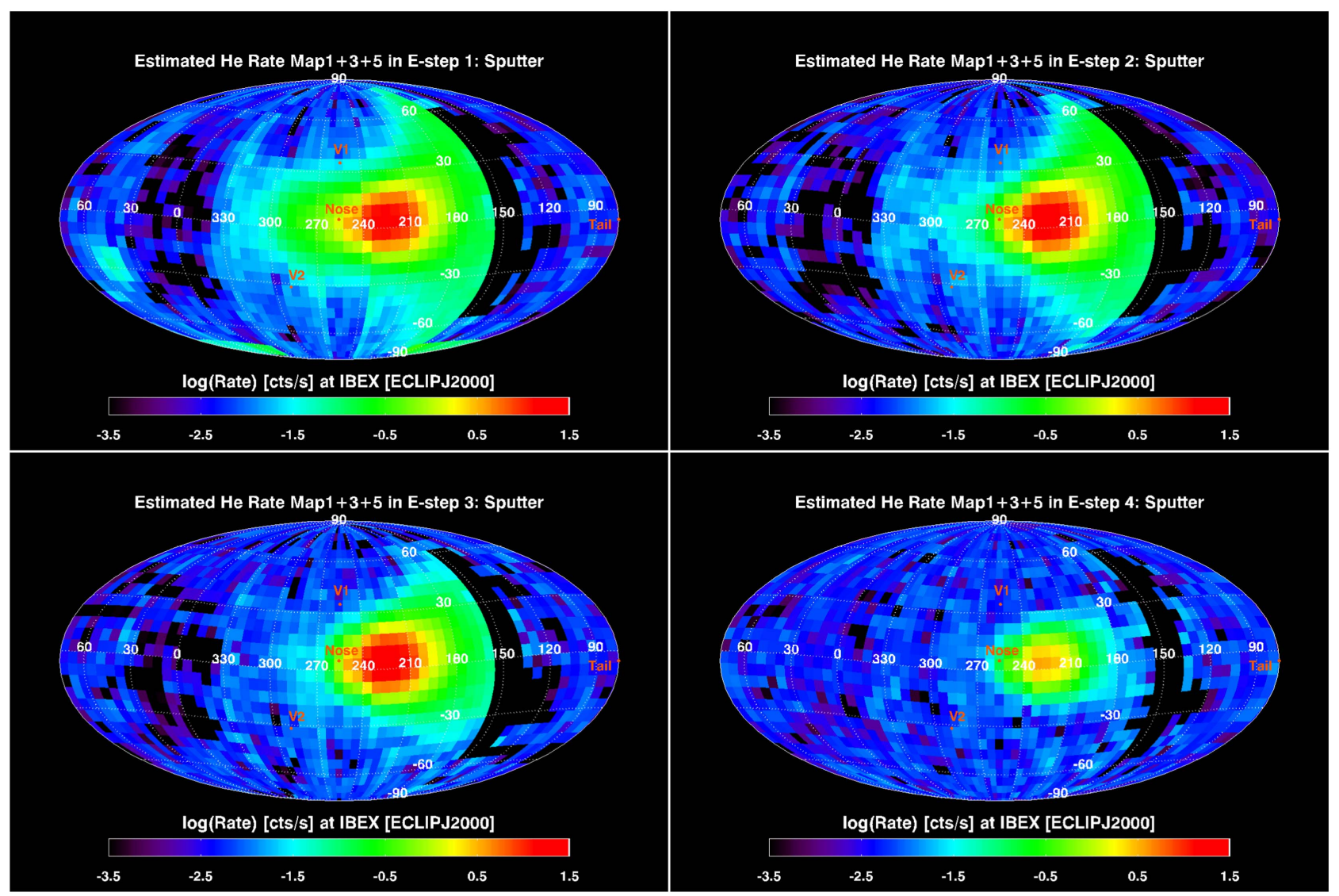

Figure 4. Sputtering-corrected He count-rate maps in E-steps 1-4 in the spacecraft reference frame. All panels use the same logarithmic color bar scaling from -3.5 to 1.5. The map projection style and legends are the same as in Figure 2.

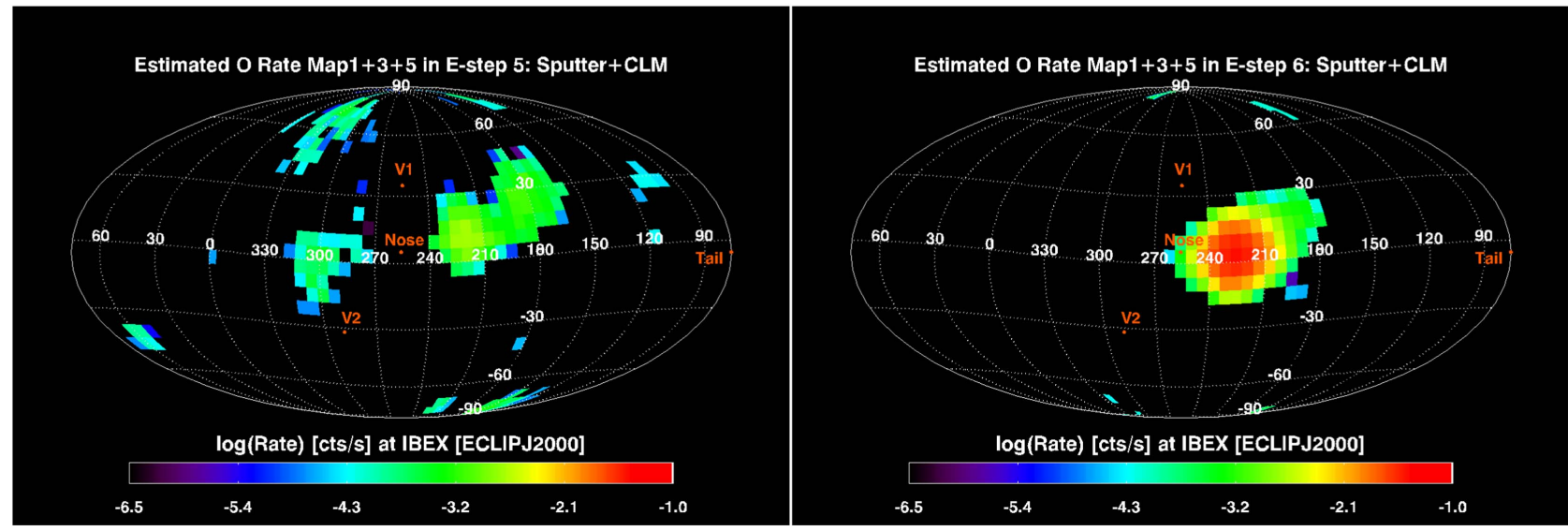

Figure 5. Sputtering-corrected $O$ count-rate maps in E-steps 5-6 in the spacecraft reference frame. Both panels use the same logarithmic color bar scaling from -6.5 to -1.0 . The map projection style and legends are the same as in Figure 2.

for the top four energy steps (E-step 5-8). Its variance is

$$
\sigma_{C, i}^{2}=\sigma_{C_{i}^{0^{-}}}^{2}+\sum_{k>i}\left(\alpha_{i k}^{\mathrm{O}^{-} \mathrm{O}}\right)^{2} \cdot \sigma_{C_{k}^{\mathrm{O}}}^{2} \cdot
$$

Then we use the confidence limit method, as modified by Park et al. (2015), to obtain the estimated O count-rate maps with an $84.13 \%$ confidence level. The median values of the upper and lower confidence limits are determined as the refined $\mathrm{O}$ count rates in E-steps 5-6. Figure 5 shows the sputtering-corrected $\mathrm{O}$ count-rate maps in E-steps 5 and 6. For E-steps 1-4, we have to subtract the sputter contribution due to the ISN He atoms. Since we concentrate on the ISN atoms and the neutral atoms derived from the ISNs in this study, the O count-rate maps in E-steps 1-4 and E-steps 7-8 are not discussed in detail here. For a more detailed description of the $\mathrm{O}$ count-rate maps, we refer the reader to the paper by Park et al. (2015).

\section{RESULTS}

Using Equations (5) and (11) we produced sputteringcorrected count-rate maps for He atoms in the energy range $15-110 \mathrm{eV}$ (E-steps 1-4) and $\mathrm{O}$ atoms in the energy range 280-600 eV (E-steps 5-6). In the sputtering-corrected count- 
Table 2

Average Background Rate $\left(\mu_{\mathrm{bg}}\right)$ and the Maximum Count Rates $\left(C_{\max }\right)$ in the Estimated He Maps in the Four Low Energy Steps with Their Standard Deviations $\left(\sigma_{\mathrm{bg}}\right.$ and $\left.\sigma_{C, \max }\right)$

\begin{tabular}{lcccc}
\hline \hline E-step & $\mu_{\mathrm{bg}}\left(\mathrm{cts} \mathrm{s}^{-1}\right)$ & $\sigma_{\mathrm{bg}}\left(\mathrm{cts} \mathrm{s}^{-1}\right)$ & $\begin{array}{c}C_{\max } \\
\left(\text { cts s}^{-1}\right)\end{array}$ & $\begin{array}{c}\sigma_{C, \max } \\
\left(\text { cts s}^{-1}\right)\end{array}$ \\
\hline 1 & $(5.12 \pm 0.12) \times 10^{-3}$ & $3.63 \times 10^{-3}$ & 18.26 & 0.10 \\
2 & $(5.28 \pm 0.12) \times 10^{-3}$ & $3.81 \times 10^{-3}$ & 20.42 & 0.11 \\
3 & $(6.28 \pm 0.12) \times 10^{-3}$ & $4.08 \times 10^{-3}$ & 21.29 & 0.11 \\
4 & $(6.35 \pm 0.09) \times 10^{-3}$ & $3.70 \times 10^{-3}$ & 3.73 & 0.05 \\
\hline
\end{tabular}

rate maps, we removed apparent signals caused by sputtering of the higher energy $\mathrm{H}$ and $\mathrm{O}$ atoms. In this section, we discuss the directional distributions of $\mathrm{He}$ and $\mathrm{O}$ atoms in these sputtering-corrected maps.

\subsection{He Maps with the Sputtering Corrections}

The corrected He maps show four features (see Figure 4). First, the red and yellow pixels between $\lambda \approx 200^{\circ}-260^{\circ}$ represent the observed directional distribution of the primary ISN He gas atoms at Earth's orbit. The maximum count rates are between 18.3 and $21.3 \mathrm{~s}^{-1}$ in E-steps $1-3$, dropping to $3.7 \mathrm{~s}^{-1}$ in E-step 4. The change of the maximum count rates in the He maps is consistent with the energy distribution of the sputtered ions (cf. Figure 1 in Möbius et al. 2012). The maximum count rates in the He maps are listed in Table 2.

Second, the ISN $\mathrm{H}$ gas atoms are still observed in the $\mathrm{He}$ map in E-step 1 as the green pixels between $\lambda=260^{\circ}$ and $300^{\circ}$. In the corrected He maps, we subtract the estimated $\mathrm{H}$ count rates from the observed $\mathrm{H}^{-}$count rates (see Equation (7)). We estimated the $\mathrm{H}$ count rates in E-steps $1-4$ via the slope of the heliospheric H ENA energy spectrum. Unlike the heliospheric $\mathrm{H}$ ENAs, however, the flux of ISN $\mathrm{H}$ atoms does not follow a power-law energy spectrum. Therefore, the ISN H emissions are not subtracted from the observed $\mathrm{H}^{-}$maps. The energy distribution of the ISN $\mathrm{H}$ atoms is complex because of the effect of the solar radiation pressure and the larger width of the $\mathrm{H}$ energy distribution. Thus, it is not simple to remove the emission due to the ISN $\mathrm{H}$ atoms from the current He maps.

Third, the green pixels between $\lambda=160^{\circ}$ and $200^{\circ}$ represent the secondary ISN He population, which is also called "Warm Breeze" (Bzowski et al. 2012; Kubiak et al. 2014, 2016). Because the logarithmic representation does not show the directional distribution of this population in detail, we compare the latitude profiles of the count rates along the latitudinal swaths between $\lambda=160^{\circ}$ and $300^{\circ}$ in the He maps. These latitude profiles are discussed in Section 4.2 in detail.

Lastly, blue and purple pixels are seen in the directions toward the heliospheric flank and tail. These pixels may represent a background contribution due to globally distributed He atoms (Grzedzielski et al. 2013). The blue and purple pixels have a low count rate $\left(<0.03 \mathrm{~s}^{-1}\right)$ and low signal-to-noise ratios $(\leqslant 3)$. Therefore, these pixels are considered as background in this study. The average intensities of these background signals are $5.1 \times 10^{-3}-6.4 \times 10^{-3} \mathrm{~s}^{-1}$. Table 2 lists the average intensities of the background contributions and their standard deviations in the He maps in E-steps 1-4. As mentioned earlier, Galli et al. (2014, 2015) examined the maximum $\mathrm{H}$ count rates caused by ISN $\mathrm{He}$ and ubiquitous background $\mathrm{H}$ count rates in the four lower energy steps of $I B E X$-Lo. They quantified the maximum $\mathrm{H}$ count rates through the measured count rates in Orbit 64 and the background through the heliospheric ENA maps in the solar inertial reference frame. The maximum count rates in the corrected $\mathrm{He}$ maps agree well with the maximum $\mathrm{H}$ count rates inferred by Galli et al. but our background levels are two times lower than the background $\mathrm{H}$ count rates examined by Galli et al. (see Tables 2 and 3 in Galli et al. 2015). There are several possible reasons to describe the roughly two times lower background levels: (1) it may indicate that the omnidirectional background in the lower energies $(<150 \mathrm{eV})$ consists of ubiquitous background $\mathrm{H}$ (Galli et al. 2014) and globally distributed $\mathrm{He}$ (Grzedzielski et al. 2013). (2) It also may be inferred that the contribution due to sputtering of heliospheric H ENAs is not noticeable in the ISN gas region (red and yellow pixels in the He maps), but strong in the background (blue and purple pixels in the He maps). In other words, the emissions in the ISN gas region mostly result from the ISN He gas. (3) Moreover, it may indicate that our extrapolation of a constant power law for heliospheric $\mathrm{H}$ to the lowest energies overestimates the fluxes of heliospheric H ENAs. Even though there are several possible reasons for the background discrepancy, it has little impact on the derivation of the primary and secondary ISN He and $\mathrm{O}$ peak since their count rates exceed the background by orders of magnitudes.

\subsection{Characteristics of the Secondary ISN He Population}

In the following, we compare the latitudinal profiles of the count rates for the swaths between $\lambda=155^{\circ}$ and $320^{\circ}$ in the corrected He maps in E-steps 1-4. These swaths include the primary ISN He gas and the secondary He population. This analysis follows the earlier work by Park et al. (2015), who looked at the latitudinal profiles of the count rates in the combined IBEX-Lo heavy neutral map in E-step 5. For each latitudinal profile, we investigate its peak in terms of ecliptic latitude, its peak width, and its peak height in count rate. We use an exponentially modified Gaussian (EMG) function to determine these parameters because the latitudinal profiles show an asymmetric distribution. We use a nonlinear least squares fit method to determine four parameters in an EMG function: peak area, peak center, peak width, and peak skewness. In this function, the inverse of the variance of the count rate is used as weight to compute the standard deviations of the four parameters. The peak latitudes and heights are estimated by fitting the curve to all count rates along the swath. Figure 6 shows two examples of the latitudinal profiles at $\lambda=162^{\circ}$ (red circles) and $216^{\circ}$ (blue triangles) in the E-step 2 He map. The red and blue curves are EMG fit curves. In the right panel of Figure 6 , the latitude profile at $\lambda=216^{\circ}$ (blue triangles) has the elevated wings that do not fit to the single EMG approximation. These wings may be due to the secondary He population, and a sum of two EMG functions could provide a more accurate result in the total strength of the signal. However, the elevated wings have little impact on the derivation of the ISN He peak because the count rates of the primary He exceed the wings by orders of magnitude. Therefore, we ignore the wing effect in this investigation.

In Figure 7(A), we plot the observed flow peak latitudes of the latitudinal profiles within the ecliptic longitude range $155^{\circ}$ $315^{\circ}$ in the corrected He maps in E-steps $1-4\left(\beta_{\text {peak }, i}(\lambda)\right)$ and the analytical model fit (yellow line; Leonard et al. 2015). The ISN He gas flow is seen in the ecliptic longitude range $205^{\circ}$ $250^{\circ}$. In this longitude range, the peak latitudes slightly 

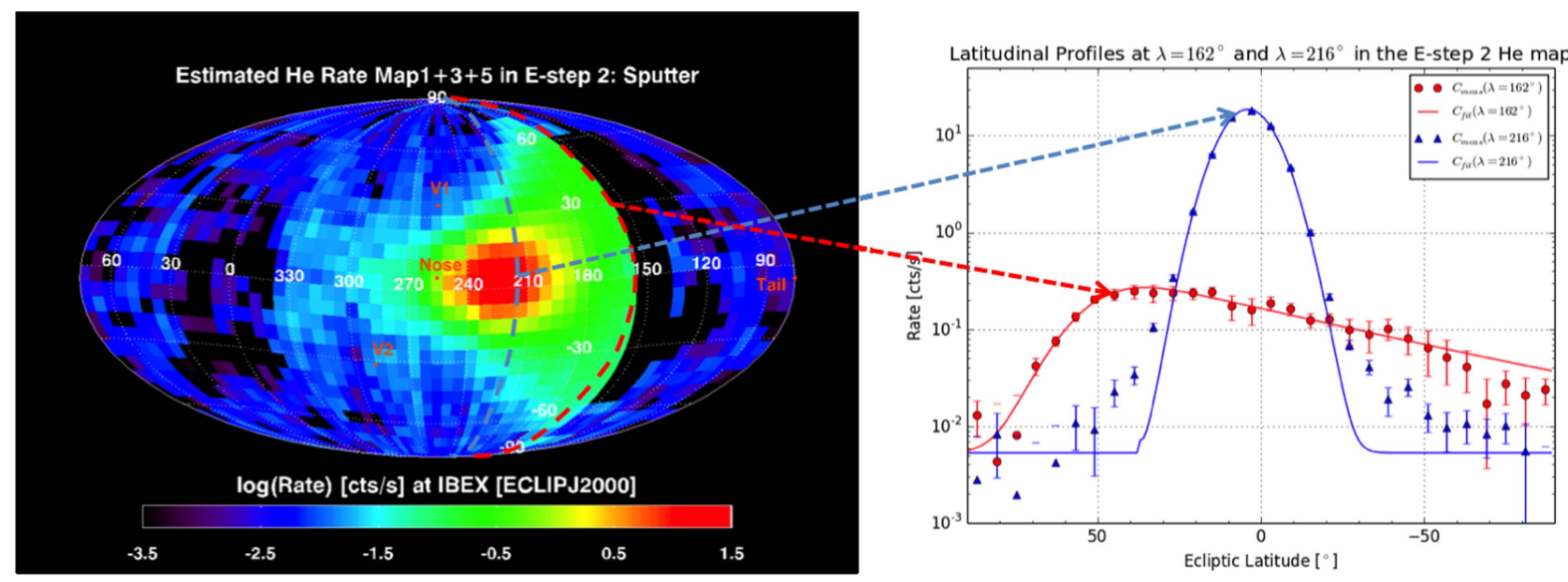

Figure 6. Left panel shows the sputtering-corrected He map in E-step 2 and the right panel illustrates two examples of the latitudinal profiles at $\lambda=162^{\circ}$ (red circles) and $216^{\circ}$ (blue triangles) in this He map. These latitudinal profiles correspond to the red and blue dashed lines in the He map.

increase from $\beta_{\text {peak }}=3^{\circ}$ to $5^{\circ}$, which is consistent with the analytical model fit. The analytical model assumes that the ISN distribution consists of a drifting Maxwellian in the local interstellar cloud with a bulk flow speed of $26.53 \mathrm{~km} \mathrm{~s}^{-1}$ and considers the Sun's gravitational field (Lee et al. 2012). Theoretically, the peak flow latitudes vary according to the flow speeds. This model is only available for a longitude range $205^{\circ}-250^{\circ}$ (Leonard et al. 2015). In the ecliptic longitude range $250^{\circ}-320^{\circ}$, the peak flow latitudes are roughly constant at $\sim 4^{\circ}$ and slightly increase to $\sim 7^{\circ}$ in E-steps $1-2$. These peak latitudes may indicate the presence of ISN $\mathrm{H}$ gas atoms or the Warm Breeze contribution as suggested by Kubiak et al. (2016). For ecliptic longitudes less than $200^{\circ}$ in the He maps, the peak latitudes sharply increase to $\sim 45^{\circ}$ in E-steps $1-2$ and $\sim 10^{\circ}$ in E-steps 3-4. These significant increases in the peak latitudes are due to the secondary ISN He population. The flow peak latitude increase is stronger in E-steps 1-2 than in E-steps $3-4$. The difference of the peak latitude variation with energy clearly indicates that the influence of the secondary ISN He population is stronger in the lower energy steps than in E-steps 3-4 because of its slow bulk speed.

In Figure 7(B), we show the flow peak widths of the latitude profiles within the same longitude range as used in Figure 7(A) in the He maps in E-steps 1-4 $\left(\sigma_{\text {peak }, i}(\lambda)\right.$ for $\lambda=155^{\circ}-315^{\circ}$ and $i=1-4$ ). There is a significant difference between the flow peak widths of the primary ISN He atoms and the secondary $\mathrm{He}$ population. As reported in the recent ISN gas papers, the flow vector of the ISN He is $\sim 75^{\circ} 7$ ecliptic inflow longitude and $\sim-5.1$ ecliptic inflow latitude (Bzowski et al. 2015; McComas et al. 2015b). In other words, the ISN gas flows to the Sun from above the ecliptic plane. Their trajectories are affected by the solar gravity. Therefore, the directional distribution of the ISN $\mathrm{He}$ atoms spreads from the nose direction $\left(255^{\circ} .7\right.$ ecliptic longitude and $+5^{\circ} .1$ ecliptic latitude) toward the northern hemisphere at $\sim 180^{\circ}$ ecliptic longitude in the sky map. In the ecliptic longitude range $\lambda=180^{\circ}-260^{\circ}$, the spread of the ISN $\mathrm{He}$ atoms is shown as an increase in the flow peak width. The flow peak width increases gradually as the ecliptic longitude decreases in the ecliptic longitude range $205^{\circ}-260^{\circ}$, but it sharply increases in the longitude range $205^{\circ}-180^{\circ}$. For longitudes less than $180^{\circ}$, the flow peak width in E-step 3 increases continually, but the peak width in energy steps $1-2$ decrease sharply. In the ecliptic longitude range $155^{\circ}-180^{\circ}$, the latitude profiles show significantly deformed, asymmetric distributions, i.e., their skewness is greater than 20 (see Figure 6). The high skewness reduces the peak width and is characteristic for the secondary ISN He population (cf., Kubiak et al. 2016). In the ecliptic longitude range $180^{\circ}-205^{\circ}$, a sharp increase of the flow peak width may represent the mixture of the primary and secondary ISN He populations. It should be noted, however, that since the distributions in E-step 4 decrease faster with longitude than in the other lower energy steps, we do not obtain reliable widths for ecliptic longitudes less than $180^{\circ}$. On the other hand, the flow peak widths increase again for ecliptic longitudes greater than $260^{\circ}$ in the He maps at the two lowest energy steps (E-steps 1 and 2). This increase may be explained by the presence of the ISN H. As mentioned earlier, the ISN $\mathrm{H}$ gas is affected by the solar radiation pressure and has a broad energy distribution due to its higher thermal speed than other heavier elements. Therefore, the ISN H gas has a broad directional distribution. Also, this increase may indicate the Warm Breeze contributions.

Figure 7(C) shows the flow peak intensities of the latitudinal profiles within the same longitude range as in Figure 7(A) from the E-steps $1-4$ He maps $\left(C_{\text {peak }, i}(\lambda)\right.$ for $\lambda=155^{\circ}-315^{\circ}$ and $i=1-4)$. The enhancement for $\lambda>260^{\circ}$ in E-step 1 is due to the ISN $\mathrm{H}$ atoms. The influence of the ISN $\mathrm{H}$ gas atoms over the ecliptic longitude range $260^{\circ}-320^{\circ}$ in E-steps 1 and 2 is also seen in Figures 7(A) and (B). In this ecliptic longitude range, the latitude profiles in E-steps 3-4 do not show a noticeable flow peak which indicates the influence of the ISN H atoms. The enhancement due to the secondary He population is revealed in the ecliptic longitude range $\lambda=160^{\circ}-205^{\circ}$. Due to the sputtering efficiency, the He map in E-step 4 has lower counting statistics by a factor of 10 compared with the He maps in E-steps 1-3. Because the parent ions of the secondary ISN He population are decelerated in front of the heliopause, the secondary He population has a lower bulk flow speed than the primary ISN He gas. In addition, the sputtered ions have a broad energy distribution below the incident energy of the parent neutral atoms. Therefore, the influence of the secondary He population is reduced in the E-step $4 \mathrm{He}$ map. In other words, the ecliptic longitude distribution of the flow peak intensities in E-step 4 may represent the primary ISN He gas distribution without any contribution from the secondary $\mathrm{He}$ population or other elements of the ISN gas flow, such as $\mathrm{H}$ and 


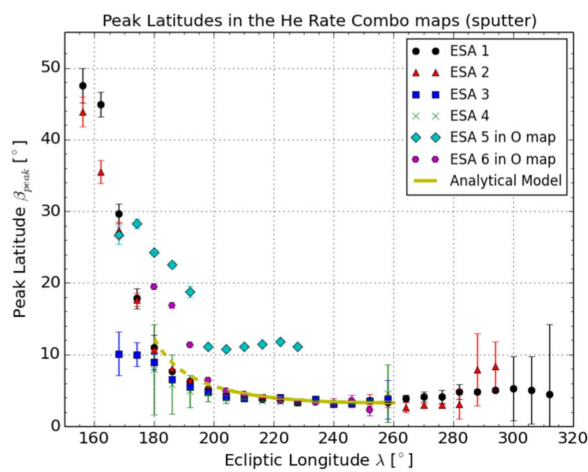

(A)

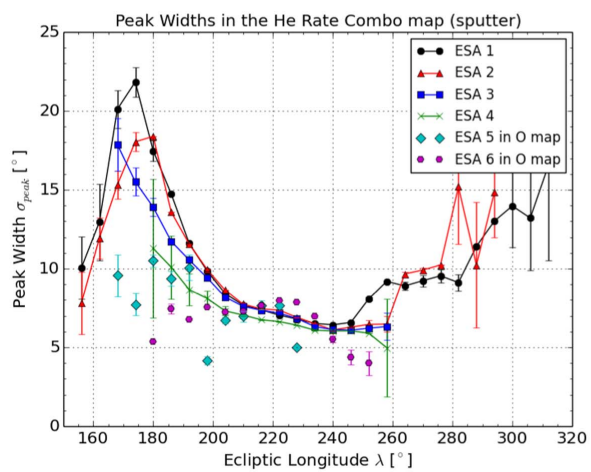

(B)

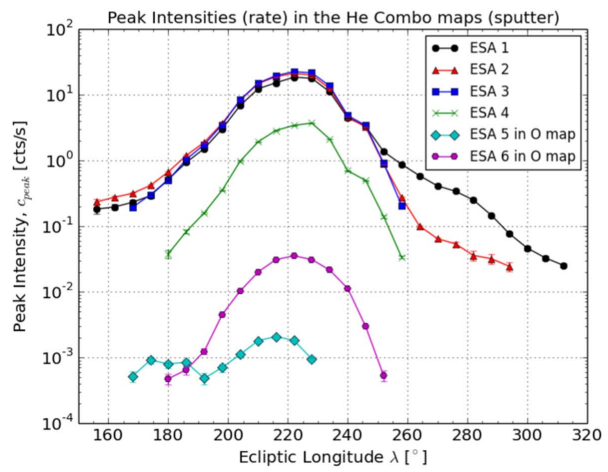

(C)

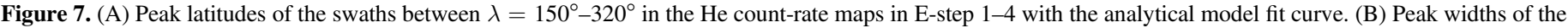
same swaths as in Panel (A) in E-steps 1-4. (C) Peak heights of the same swaths with Panel (A) in E-steps 1-4.

O. The separation of the primary ISN He gas and the secondary He population will be discussed in detail in Section 5.1.

\subsection{Sputtering-corrected $O$ Maps}

Park et al. (2015) investigated the $\mathrm{O}$ maps produced from the $I B E X$-Lo observations using statistical methods, but they did not apply the sputtering corrections to the maps. These methods showed that the primary ISN $\mathrm{O}$ and $\mathrm{Ne}$ gas is present from $200^{\circ}$ to $250^{\circ}$ ecliptic longitude in the $\mathrm{O}$ maps in E-steps 5 and 6 . They also showed that an extended tail emission is seen in the ecliptic longitude range $180^{\circ}-200^{\circ}$. In our study, we corrected the $\mathrm{O}$ maps for the sputtering from higher energies and then applied the confidence limit method as used by Park et al. (2015). Figure 5 shows the sputtering-corrected $\mathrm{O}$ maps in E-steps 5 and 6.

In the right panel of Figure 5, the primary $\mathrm{O}$ and $\mathrm{Ne}$ gas is clearly seen in the longitude range $200^{\circ}-250^{\circ}$ in the sputteringcorrected O map in E-step 6. Its tail spreads toward the higher latitude and lower ecliptic longitude, which is consistent with results obtained in previous studies (Möbius et al. 2009; Park et al. 2015). However, the $\mathrm{O}$ map in E-step 5 shows a somewhat different directional distribution of neutral atoms (left panel of Figure 5). In the O map in E-step 5, the apparent emissions caused by sputtering from higher energy $\mathrm{O}$ atoms are removed by the sputtering correction. Most of the higher energy $\mathrm{O}$ atoms are the ISN $\mathrm{O}$ atoms, which have a bulk energy of $527 \mathrm{eV}$ at Earth's orbit in the observer reference frame. After the sputtering correction, a remnant emission is seen in the ecliptic longitude range $165^{\circ}-195^{\circ}$. It is reasonable to assume that this remnant emission represents the secondary ISN O population, which has a different velocity distribution from the primary ISN O gas. Although the directional distribution of the secondary $\mathrm{O}$ population is somewhat shifted to the lower ecliptic longitudes than the extended tail referred to by Park et al. (2015), these two emissions seem to be caused by the same $\mathrm{O}$ population. A possible reason for the different directional distribution between the secondary $\mathrm{O}$ signals in the two energy steps is discussed in Section 5.2.

Here we note two things. (1) There is energy loss on the conversion surface when an incoming neutral atom is converted to a negative ion (Fuselier et al. 2009), and (2) the apparent energy of a neutral atom in the spacecraft reference frame depends on its incidence angle (McComas et al. 2010). These two things are important to understand the $\mathrm{O}$ atoms observed in E-step 5. For instance, the apparent energy of an $\mathrm{O}$ atom with
$0 \mathrm{~km} \mathrm{~s}^{-1}$ at 150 au from the Sun is $422 \mathrm{eV}$ in the spacecraft frame at 1 au when the spacecraft is moving toward the atom's incoming direction. It seems that the atom should be observed in E-step 6 instead of E-step 5 because the energy acceptance of $422 \mathrm{eV}$ is $\sim 0.3$ for E-step 5 (see Figure 1). However, since there is approximately $15 \%$ energy loss on the conversion surface (Fuselier et al. 2009), the energy of the converted $\mathrm{O}^{-}$ ion is reduced to $\sim 359 \mathrm{eV}$. In addition, the apparent energy of an incoming atom decreases as its incidence angle is deviated from the anti-parallel direction of the Earth's velocity vector. For the $\mathrm{O}$ atoms with the incidence angle range of $40^{\circ}-80^{\circ}$ (i.e., $+50^{\circ}-+10^{\circ}$ in the ecliptic latitude), their apparent energies should be lower than $422 \mathrm{eV}$. Due to the energy loss on the conversion surface, their converted $\mathrm{O}^{-}$ions should have energies lower than $359 \mathrm{eV}$ and the energy acceptance for $359 \mathrm{eV}$ is greater than 0.5 in E-step 5. Therefore, it is reasonable to conclude that the $\mathrm{O}$ atoms with $0 \mathrm{~km} \mathrm{~s}^{-1}$ at 150 au can be observed in E-step 5 at Earth's orbit. We can perform the same exercise with the $\mathrm{O}$ atom with $26 \mathrm{~km} \mathrm{~s}^{-1}$ at $150 \mathrm{au}$ and the apparent energy of its converted ion is less than and equal to $436 \mathrm{eV}$. Since the energy acceptance of $436 \mathrm{eV}$ is not zero in E-step 5, there is a possibility to measure the $\mathrm{O}$ atom in E-step 5.

The latitudinal profiles obtained from the $\mathrm{O}$ maps reveal the secondary $\mathrm{O}$ features more clearly. As can be seen in Figure 7(A), the increase of the flow peak latitude in the $O$ maps is steeper than in the He maps for ecliptic longitudes less than $200^{\circ}$. In Figure $7(\mathrm{C})$, the flow peak intensities related to the secondary $\mathrm{O}$ population are seen in the longitude range $166^{\circ}$ to $192^{\circ}$, which separates them clearly from the peak intensities of the primary ISN $\mathrm{O}$ gas.

In the left panel of Figure 5, we can also see an emission in the ecliptic longitude range $195^{\circ}-230^{\circ}$, which may be a remnant of the primary $\mathrm{O}$ and $\mathrm{Ne}$ gas flow. As can be seen in Equation (11), for the sputtering correction in the O map, we only consider the contribution due to the primary ISN O atoms in E-step 5. However, the IBEX-Lo observations show that there is a considerable fraction of the ISN Ne atoms at Earth's orbit (Bochsler et al. 2012; Park et al. 2014). When these Ne atoms come into IBEX-Lo, they sputter $\mathrm{C}^{-}$and $\mathrm{O}^{-}$ions from the conversion surface and then these negative ions are registered as " $\mathrm{O}^{-}$" events. Because both $\mathrm{O}$ and $\mathrm{Ne}$ atoms are recorded as " $\mathrm{O}^{-}$" events in $I B E X$ - $\mathrm{Lo}$, it is impossible to unambiguously distinguish the " $\mathrm{O}^{-}$" events caused by the ISN $\mathrm{Ne}$ atoms from the genuine " $\mathrm{O}^{-}$" events. Therefore, we do not consider the contribution of the ISN Ne atoms in this study. 
(A)

A) Normalized Peak Intensities in the He combo maps

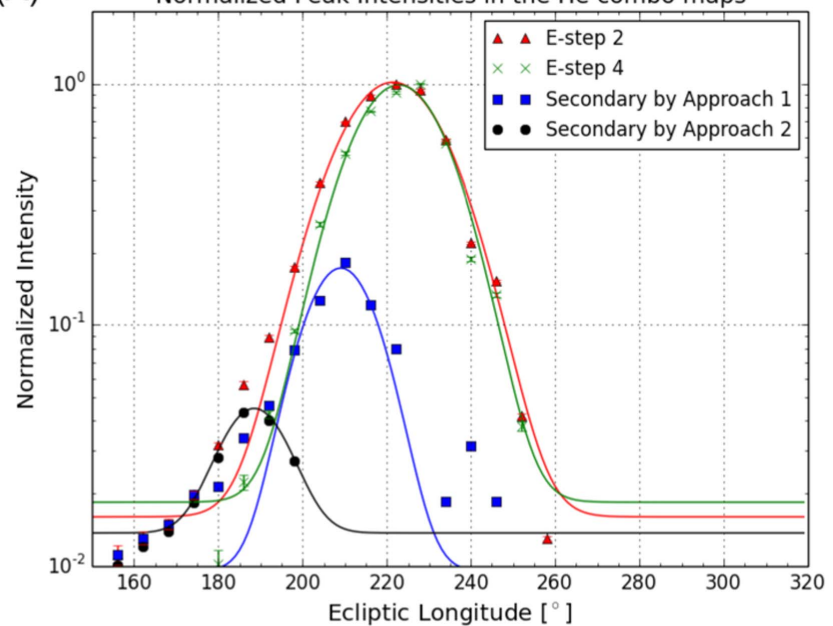

(B)

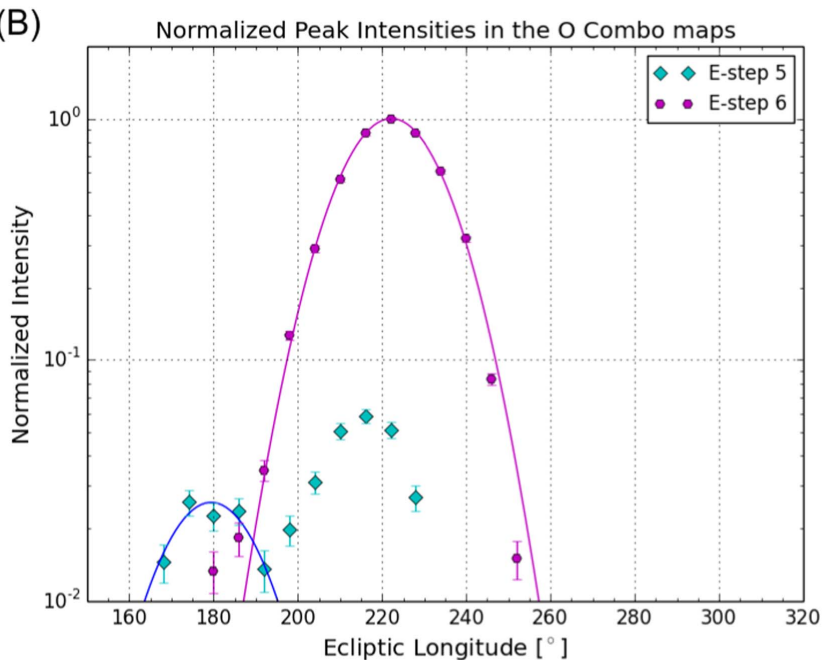

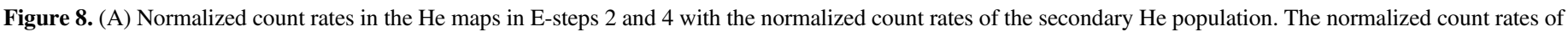

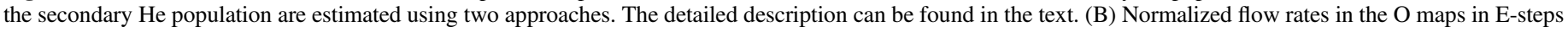
5 and 6.

\section{DISCUSSION}

As discussed in Section 3.2, neutral He atoms are observed as sputtered $\mathrm{H}^{-}$ions with a broad energy distribution below their incident energy. We expect that the secondary ISN He atoms have lower bulk energies than the primary ISN He gas. Therefore, IBEX-Lo samples not only the primary ISN He atoms but also the secondary He population in the energy ranges $15-110 \mathrm{eV}$ (E-steps 1-4). The two He components have two different bulk energies, which lead to different energy distributions of sputtered ions. These different energy distributions are represented as different ratios among the detected portions of sputtered $\mathrm{H}^{-}, \mathrm{C}^{-}$, and $\mathrm{O}^{-}$ions in the TOF spectrum (Wurz et al. 2008; Park et al. 2014). However, this difference is subtle, and we cannot untangle the mixture of the two components without modeling the observations as a mix of the two components. As discussed in Section 4.3, in contrast to $\mathrm{He}$, the emission due to the secondary $\mathrm{O}$ population in the $\mathrm{O}$ maps is clearly separated from that of the primary ISN O gas. In this section, we consider two approaches with simple Gaussian distributions to separate the secondary He population from the primary ISN He atoms. Then we compare the resulting peak longitudes of the secondary $\mathrm{He}$ and $\mathrm{O}$ populations. In addition, we discuss the possible flow speed and longitude ranges for the secondary populations.

\subsection{Peak Longitude of the Secondary He Population}

As discussed in Section 4.2, it is reasonable to assume that the contribution of the secondary He population is minimized in the E-step $4 \mathrm{He}$ map unless the bulk energy of the secondary He population is as high as that of the primary ISN He gas. In fact, the secondary $\mathrm{He}$ component created in the outer heliosheath occurs at lower energies through charge exchange interactions between the ISN atoms and interstellar ions. Therefore, we can assume that the peak count rates in E-step 4 (the green " $\mathrm{x}$ " markers in Figure 7(C)) represents the count rates of the primary ISN He gas as a function of the ecliptic longitude of the sensor boresight. Further, the peak count rates in the lower energy steps (E-steps 1-3) are regarded as due to a mixture of the primary ISN and secondary He populations. To obtain the count rates of the secondary He population, we carry out the following procedure. First, we normalize the count rates in E-steps 1-4 to their maximum count rates $\left(R_{\text {peak }, i}(\lambda)=C_{\text {peak }, i}(\lambda) / C_{\text {max }, i}\right.$, where $R_{\text {peak }, i}(\lambda)$ and $C_{\text {max }, i}$ are a normalized count rate at $\lambda$ and a maximum count rate in E-step $i$, respectively). Then we subtract the normalized count rates in E-step 4 from the normalized count rates in E-steps $1-3$, respectively $\left(R_{\text {rem }, i}(\lambda)=R_{\text {peak }, i}(\lambda)-R_{\text {peak }, i=4}(\lambda)\right.$ for $i=1,2$, and 3). The residuals of the normalized count rates $\left(R_{\mathrm{rem}, i}(\lambda)\right)$ should represent the secondary He population. In the next step, we determine the peak longitudes, their uncertainties, and their widths $( \pm 1 \sigma$ from the flow peak longitude) in a least-squares fit of the residuals to Gaussians. In Figure 8(A), we plot the normalized count rates in E-steps 2 $\left(R_{\text {peak }, i=2}(\lambda)\right.$, red triangles $)$ and $4\left(R_{\text {peak }, i=4}(\lambda)\right.$, green crosses $)$ with their Gaussian fit curves (red and green lines, respectively). The blue squares are the normalized count rates of the secondary He population in E-step $2\left(R_{\mathrm{rem}, i=2}(\lambda)\right)$. Based on this approach (Approach 1), the estimated peak longitudes and widths of both the primary ISN and the secondary $\mathrm{He}$ populations are compiled in the top panel of Table 3 . The weighted mean values of the flow peak longitude and width for the secondary He population are $209^{\circ} .0 \pm 0.8$ and $7^{\circ} .6 \pm 0.9$, respectively.

We also used an alternate approach (Approach 2) to identify the secondary He population in the IBEX-Lo observations. Figure 7(C) shows that the flow rates in E-steps 1-3 are very similar. For the normalized count rates in E-steps $1-3$, the average peak longitude and its width are $221.4 \pm 0.2$ and $12^{\circ} .0 \pm 0.2$, respectively (top panel of Table 3). In Approach 2, we assume that the Gaussian distribution with the average peak longitude and its width represents the primary ISN He gas $\left(R_{\text {pri, }}\right.$ fit $(\lambda)$ ). Then we subtract the estimated count rates of the primary ISN He gas from the normalized count rates in E-step 2 assuming that the residuals may represent the secondary $\mathrm{He}$ population $\left(R_{\text {rem }}(\lambda)=R_{\text {peak }, i=2}(\lambda)-R_{\text {pri, fit }}(\lambda)\right)$. We plot the residual count rates as the black circles in Figure 8(A). In the second approach, the estimated peak longitude and its width are $187^{\circ} .9 \pm 0.2$ and $7^{\circ} .0 \pm 0.3$, respectively. The peak longitude of the secondary He population is different by $\sim 20^{\circ}$ from the peak location obtained in the first approach. This difference may be due to the use of different energy steps. As discussed 
Table 3

Flow Peak Longitude and Width of the Primary and Secondary ISN He (top) and $\mathrm{O}$ (bottom) Populations as Obtained from the He and O Maps

\begin{tabular}{|c|c|c|c|c|}
\hline \multicolumn{5}{|l|}{ He Map } \\
\hline \multirow{2}{*}{ ESA } & \multicolumn{2}{|c|}{ Primary } & \multicolumn{2}{|c|}{ Secondary } \\
\hline & Peak & Width & Peak & Width \\
\hline 4 & $222^{\circ} 8 \pm 0.5$ & $10^{\circ} 6 \pm 0.6$ & $\ldots$ & $\ldots$ \\
\hline 3 & $221.5 \pm 0.4$ & $11.8 \pm 0.5$ & $209.6 \pm 1.3$ & $8.0 \pm 1.5$ \\
\hline 2 & $221^{\circ} 1 \pm 0.3$ & $12.0 \pm 0.3$ & $209.1 \pm 1.2$ & $7.8 \pm 1.4$ \\
\hline 1 & $221^{\circ} .7 \pm 0.3$ & $12^{\circ} 1 \pm 0.3$ & $207.8 \pm 1.7$ & $6.6 \pm 1.8$ \\
\hline Approach 1 & $222.8 \pm 0.5$ & $10^{\circ} 6 \pm 0.6$ & $209^{\circ} 0 \pm 0.8$ & $7.6 \pm 0.9$ \\
\hline Approach 2 & $221^{\circ} .4 \pm 0.2$ & $12.0 \pm 0.2$ & $187^{\circ} .9 \pm 0.2$ & $7^{\circ} .0 \pm 0.3$ \\
\hline \multicolumn{5}{|l|}{ O Map } \\
\hline \multirow[t]{2}{*}{ ESA } & \multicolumn{2}{|c|}{ Primary } & \multicolumn{2}{|c|}{ Secondary } \\
\hline & Peak & Width & Peak & Width \\
\hline 5 & $\ldots$ & $\ldots$ & $179.4 \pm 1.4$ & $11.5 \pm 2.2$ \\
\hline 6 & $222^{\circ} .2 \pm 0.2$ & $11.5 \pm 0.2$ & $\ldots$ & $\ldots$ \\
\hline ISN O & $222^{\circ} .2 \pm 0.2$ & $11.5 \pm 0.2$ & $179^{\circ} .4 \pm 1.4$ & $11.5 \pm 2.2$ \\
\hline
\end{tabular}

above, the observed count rates in E-step 4 likely contain solely primary ISN He atoms because we assume that the secondary $\mathrm{He}$ population arrives with noticeably lower energies. In E-steps 1-3, the secondary He population likely contributes to the main peak on its lower longitude flank, which leads to a shift and broadening of the Gaussian fit. Therefore, in Approach 2, we likely include part of the secondary $\mathrm{He}$ population in the subtraction, which results in a shift of the peak to lower longitudes. If we were to correct for this effect, the peak longitude should be shifted to higher longitudes.

\subsection{Peak Longitude of the Secondary O Population}

Based on the assumption that a Gaussian distribution represents the longitude distribution of the normalized peak intensities of each latitude profile, we conducted a rough quantitative analysis to determine the count-rate peak locations of the two observed $\mathrm{O}$ populations in ecliptic longitude. Similar to the method used in Section 5.1, we normalized the peak intensities of the latitude profiles in the E-step 5-6 O maps to the maximum $\mathrm{O}$ count rate in E-step $6\left(R_{\text {peak }, i}^{\mathrm{O}}(\lambda)=C_{\text {peak }, i}^{\mathrm{O}}\right.$ $\left.(\lambda) / C_{\max , i=6}^{\mathrm{O}}\right)$. We show these normalized peak intensities in Figure $8(\mathrm{~B})$ as cyan diamonds for the E-step $5 \mathrm{O}$ map and purple circles for the E-step $6 \mathrm{O}$ map. We then deduced a peak longitude and its width from a least-squares fit of the normalized peak intensities in latitude to a Gaussian distribution. Figure 8(B) includes the Gaussian fit curve for the secondary $\mathrm{O}$ population as the blue solid line and the fit curve for the primary ISN $\mathrm{O}$ gas as the purple solid line. The longitudes and widths of the peak are listed in the bottom panel of Table 3. In the observer frame, the peak of the primary ISN $\mathrm{O}$ gas is at $222^{\circ} .2$ ecliptic longitude and its width is 11.5 . The peak longitude of the secondary $\mathrm{O}$ population is $179^{\circ} .4 \pm 1.4$ and its width is $11.5 \pm 2.2$, which is approximately $40^{\circ}$ away from the peak of the primary ISN O gas in ecliptic longitude. The intensity of the secondary $\mathrm{O}$ population is approximately $2.5 \%$ of the maximum rate of the primary ISN $\mathrm{O}$ gas.
The peak longitude of the secondary $\mathrm{O}$ population differs by approximately $10^{\circ}$ from the value obtained in the previous study, which relied on an $\mathrm{O}$ map without the sputtering correction (Park et al. 2015). As discussed in Park et al. (2015), the directional distribution of $\mathrm{O}$ atoms in the energy range $280-600 \mathrm{eV}$ has a tail toward higher latitude and lower longitude $\left(\mathrm{O}^{-}\right.$maps in E-steps $5-6$ in Figure 3). This tail is also seen in the sputtering-corrected $O$ map in E-step 6 (Figure 5(B)). In Figure 8(B), we also show the Gaussian fit curve of the normalized flow peak intensities for the primary ISN $\mathrm{O}$ gas. The two data points at $\lambda=180^{\circ}$ and $186^{\circ}$ (purple circles) fall above the fit curve and thus correspond to the tail. It is reasonable to assume that the tail in E-step 6 is composed of a mixture of the primary ISN $\mathrm{O}$ atoms and the secondary $\mathrm{O}$ population. The sputtering corrections cannot distinguish between the intrinsic sources of the tail emissions, and thus may also remove the sputter contribution of the tail from the measured $\mathrm{O}$ emissions in E-step 5. Therefore, the peak intensities at $\lambda=180^{\circ}$ and $186^{\circ}$ in E-step 5 may be underestimated. In other words, part of the secondary $\mathrm{O}$ population close to the primary ISN O flow peak may also be subtracted. Due to the underestimated peak intensities in E-step 5 , the apparent peak longitude of the secondary $\mathrm{O}$ population may be shifted to lower longitude compared to the actual distribution. Therefore, the actual peak longitude of the secondary $\mathrm{O}$ population in the $\mathrm{O}$ maps is likely larger than $179^{\circ}$.

\subsection{Possible Flow Speed and Longitude Ranges for the Secondary Populations}

The IBEX science team suggested good "working values" of the primary ISN He gas flow vector for the community to use. The working values are $V_{\mathrm{ISM} \infty} \sim 25.4 \mathrm{~km} \mathrm{~s}^{-1}, \lambda_{\mathrm{ISM} \infty} \sim 75^{\circ} .7$, $\beta_{\mathrm{ISM} \infty} \sim-5^{\circ} .1$, and $T_{\mathrm{He} \infty} \sim 7500 \mathrm{~K}$ at $\sim 1000$ au upstream (McComas et al. 2015b). In the Sun's gravitational field, the incoming interstellar atoms travel along the hyperbolic trajectories. IBEX samples interstellar atoms near the perihelia of their trajectories. These observations are independent of the ISN gas flow direction at infinity. Due to the orientation of the $I B E X$ spin axis toward the Sun, IBEX observations provide interstellar atom distributions at 1 au in a plane approximately perpendicular to the Earth-Sun line. These distributions are equivalent to the latitudinal profiles. For a hyperbolic trajectory, the equation for the true anomaly at infinity $\theta_{\infty}$ provides the relation between the observer longitude $\lambda_{\text {Obs }}$ of the ISN bulk flow perihelion at 1 au and the ISN flow speed $V_{\infty}$ and longitude $\lambda_{\infty}$ at infinity (Lee et al. 2012; Möbius et al. 2012):

$$
\begin{aligned}
& V_{\infty}=\sqrt{\left(\frac{\mathrm{GM}_{s}}{r_{\mathrm{E}}}\right) \cdot\left(\frac{-1}{\cos \theta_{\infty}}-1\right)}, \text { where } \\
& \theta_{\infty}=\lambda_{\infty}+180^{\circ}-\lambda_{\text {Obs }},
\end{aligned}
$$

In Equation (13), the observer longitude $\lambda_{\text {Obs }}$ of the ISN bulk flow perihelion at 1 au is equivalent to the $I B E X$ location for the observation of interstellar atoms at the perihelia of their trajectories relative to $0^{\circ}$ ecliptic longitude. The true anomaly at infinity $\theta_{\infty}$ is an angle between the velocity vector at infinity and the space vector at Earth's orbit. Due to the sensor viewing direction being perpendicular to the spin axis, the observer 


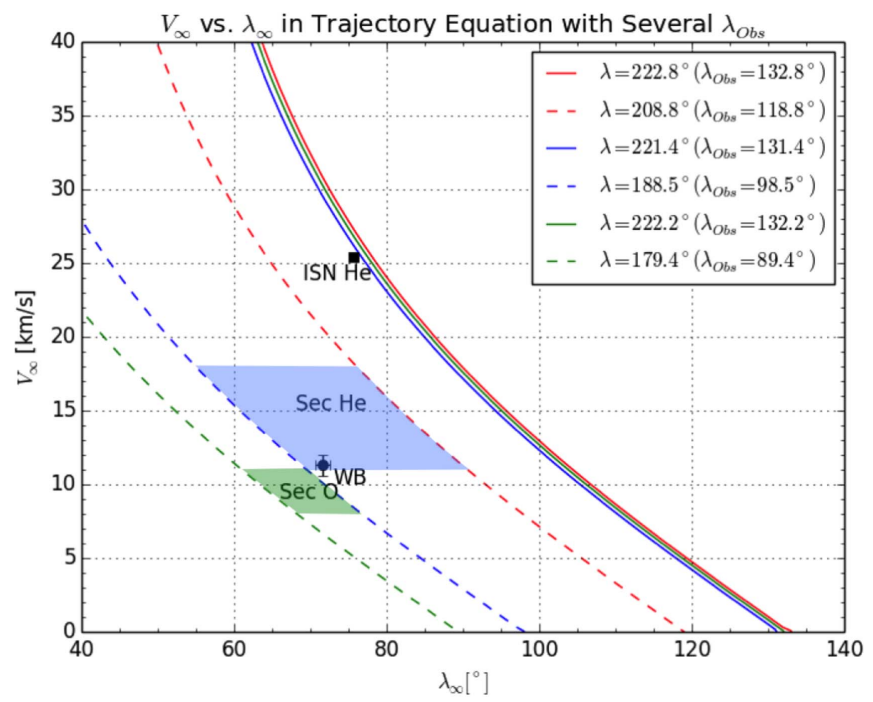

Figure 9. $V_{\infty}-\lambda_{\infty}$ relations for several observer longitudes at Earth's orbit. For the primary ISN He gas, the flow peak longitudes are $223^{\circ}$ (red solid line) in Approach 1 and $221^{\circ}$ (blue solid line) in Approach 2. For the secondary He population, the flow peak longitude is $209^{\circ}$ in Approach 1 (red dashed line) and $188^{\circ}$ in Approach 2 (blue dashed line). The blue shadow represents the possible $V_{\infty}-\lambda_{\infty}$ combinations for secondary ISN He. The flow peak longitude of primary ISN $\mathrm{O}$ is $222^{\circ}$ (green solid line) and that of secondary $\mathrm{O}$ is $179^{\circ}$ (green dashed line). The green shadow indicates the possible $V_{\infty}-\lambda_{\infty}$ combinations for secondary $\mathrm{O}$. Also shown are the suggested combination for the primary ISN He flow ( $V_{\infty, \text { ISNHe }}, \lambda_{\infty, \text { ISNHe }}$; black square; McComas et al. 2015b) and for the Warm Breeze $\left(V_{\infty, W B}, \lambda_{\infty, W B}\right.$; black circle; Kubiak et al. 2016).

longitude is shifted $90^{\circ}$ from the sensor viewing direction ( $\lambda_{\text {Obs }}=\lambda-90^{\circ}$ for the ram). For the working value of the primary ISN flow, the ecliptic longitude of the sensor viewing direction $(\lambda)$ for the maximum ISN flow observation is $220^{\circ} .4$ $\left(\lambda_{\text {Obs }}=130^{\circ} .4\right)$, which is used as a reference in this section.

In Section 5.1, we have estimated the flow peak longitudes of the primary ISN He gas and the secondary He population using two approaches. According to the relation between the observer longitude and the ecliptic longitude of the sensor viewing direction, the observer peak longitudes of the primary ISN He gas are $132^{\circ} .8$ for Approach 1 and $131^{\circ} .4$ for Approach 2. Using Equation (13), we derive the relations between $V_{\infty}$ and $\lambda_{\infty}$ for these observer longitudes, which are illustrated by the red and blue solid lines in Figure 9, respectively. These observer longitudes estimated using two approaches are slightly larger than the reference observer longitude $\left(\lambda_{\text {Obs }}=130^{\circ} .4\right)$. However, this slight mismatch may be acceptable because we do not correct for the reduction in flux due to ionization processes, such as photoionization by solar EUV, charge exchange with the solar wind, and electron impact ionization. This reduction increases with the true anomaly at infinity $\theta_{\infty}$ (Möbius et al. 2012). For a neutral particle with a faster speed, the trajectory is less deflected and its perihelion is at a larger longitude than that of a neutral particle with a slower speed. This bias shifts the observed peak longitude to a larger longitude. For the analysis of the ISN flow peak, this bias is crucial, but it is insignificant compared with the uncertainties in the directional distributions of the secondary neutral atoms.

For the secondary He population, the observed longitudes are $119^{\circ} .0 \pm 0.8$ for Approach 1 and $97^{\circ} .9 \pm 0.2$ for Approach
2. In Figure 9, the $V_{\infty}-\lambda_{\infty}$ relations for the secondary $\mathrm{He}$ populations are represented as red and blue dashed lines, respectively. As discussed in Section 4.1, the final peak longitude of the secondary He population may fall between the two peak longitudes derived from the different approaches, depending on how much each of the approaches is affected systematically. Therefore, the exact $V_{\infty}-\lambda_{\infty}$ relation of the secondary $\mathrm{He}$ population may be located between the two dashed lines (red and blue) in Figure 9. For O, the observer longitudes are $132^{\circ} .2 \pm 0.2$ for the primary ISN $\mathrm{O}$ and $89^{\circ} .4 \pm 1.4$ for the secondary $\mathrm{O}$ population. The $V_{\infty}-\lambda_{\infty}$ relations for the primary ISN $\mathrm{O}$ and secondary $\mathrm{O}$ populations are indicated by the green solid and dashed lines, respectively. As mentioned in Section 4.2, our estimate of the observer longitude of the secondary $\mathrm{O}$ population has moved $\sim 10^{\circ}$ toward lower longitude over the value reported in Park et al. (2015) due to the subtraction of a part of the secondary $\mathrm{O}$ population close to the primary ISN O flow. As a consequence, the exact $V_{\infty}-\lambda_{\infty}$ relation of the secondary $\mathrm{O}$ population should be located between the green dashed line $\left(\lambda=179^{\circ}\right)$ and the blue dashed line $\left(\lambda=189^{\circ}\right)$.

Now we consider the secondary ISN population hypothesis. Secondary ISN populations are created by charge exchange between interstellar plasma and ISN atoms. For He, Bzowski et al. (2012) showed that the $\mathrm{He}^{+}+\mathrm{He}$ charge exchange reaction dominates the production of secondary He in the outer heliosheath:

$$
\mathrm{He}+\mathrm{He}^{+} \rightarrow \mathrm{He}^{+}+\mathrm{He}_{\mathrm{sec}} .
$$

For $\mathrm{O}$, the relevant charge exchange reaction in the outer heliosheath is

$$
\mathrm{H}+\mathrm{O}^{+} \rightarrow \mathrm{H}^{+}+\mathrm{O}_{\text {sec }}
$$

According to a global heliospheric model by Izmodenov et al. (2005), the typical plasma bulk speed is $\sim 4 \mathrm{~km} \mathrm{~s}^{-1}$ Sunward along the upwind direction in the outer heliosheath. As an estimate of the typical speeds of the secondary populations in the outer heliosheath, we take the sum of the plasma bulk and thermal speed:

$$
V_{\mathrm{sec}, \infty}=V_{\mathrm{B}, \text { plasma }}+V_{\mathrm{th}},
$$

where $V_{\mathrm{th}}=\sqrt{8 k T / \pi m}$ is a thermal speed. Izmodenov et al. (2005) also stated that the plasma temperature increases to $\sim 35,000 \mathrm{~K}$ in the outer heliosheath, and Kubiak et al. (2016) estimated a Warm Breeze temperature of $\sim 9500 \mathrm{~K}$ based on the $I B E X$ observations. For these two temperatures, the typical speeds of the secondary He population in the outer heliosheath would be approximately $18 \mathrm{~km} \mathrm{~s}^{-1}$ and $11 \mathrm{~km} \mathrm{~s}^{-1}$, respectively. With the same assumptions, the estimated bulk speed of the secondary $\mathrm{O}$ population would be $\sim 11 \mathrm{~km} \mathrm{~s}^{-1}$ for $T_{\text {plasma }} \sim 35,000 \mathrm{~K}$ and $\sim 8 \mathrm{~km} \mathrm{~s}^{-1}$ for $T_{\text {plasma }} \sim 9500 \mathrm{~K}$. Using these estimated flow speeds at infinity and the observer longitudes at Earth's orbit, we traced the observed maxima of these flow components back to the heliopause through Equation (13). Based on the observer longitudes for the observation of the maximum secondary populations at Earth's orbit, discussed in Section 4.2 for He and in Section 4.3 for $\mathrm{O}$, we have deduced secondary flow longitudes beyond the heliopause as $\lambda_{\infty, \mathrm{Sec}, \mathrm{He}}=55^{\circ}-88^{\circ}$ for $\mathrm{He}$ and $\lambda_{\infty, \mathrm{Sec}, \mathrm{O}}=61^{\circ}-77^{\circ}$ for $\mathrm{O}$. 
In Figure 9, the blue and green shaded areas represent possible ranges of the flow longitudes and speeds for the secondary He (blue) and $\mathrm{O}$ (green) components beyond the heliopause, respectively. If the hypothesis of secondary ISN populations is correct, these results support the following ideas. First, the possible flow longitudes of the secondary populations are mostly lower than the flow longitude of the primary ISN He gas $\left(\lambda_{\mathrm{ISM} \infty}=75^{\circ} .7\right)$. This result may support the hypothesis of asymmetry of the outer heliosheath as discussed by Zieger et al. (2013) and Möbius et al. (2015b). Zieger et al. indicated that current estimates of the interstellar magnetic field direction lead to a spatially confined quasi-parallel slow bow shock. The slow bow shock model predicts that the starboard flank of the heliospheric boundary is thicker than the port flank. Therefore, the secondary ISN components on the starboard side of the heliosphere may be stronger than those from the port and nose direction.

Second, the distributions of the secondary $\mathrm{O}$ is narrower than the distribution of secondary $\mathrm{He}$ in flow speed. This result is consistent with the interstellar $\mathrm{He}^{+}$ion population having a higher thermal speed than interstellar $\mathrm{O}^{+}$ions in the outer heliosheath, which can be linked to the different masses of the two species.

In Figure 9, we also denote the suggested flow longitudes and speeds of the ISN He gas (black square; McComas et al. 2015b) and the Warm Breeze (black circle; Kubiak et al. 2016) at infinity. Kubiak et al. (2016) analyzed the IBEX-Lo observations with E-step 2 during the early ISN observation seasons 2010-2014 (from mid-November to mid-January of each of those years) and unlike us, assumed that the entire observed signal is due to helium atoms. Using the Warsaw Test Particle model (Sokół et al. 2015), they simulated a superposition of neutral He fluxes at Earth's orbit originating from two Maxwell-Boltzmann populations of $\mathrm{He}$ atoms in the outer heliosheath. They derived the flow parameters of the Warm Breeze through the parameter-fitting method by Swaczyna et al. (2015). The flow longitude and speed of the Warm Breeze they inferred are $\lambda_{\infty, \mathrm{Sec}, \mathrm{He}}=71.6$ and $V_{\infty, \mathrm{Sec}, \mathrm{He}}=11.3 \mathrm{~km} \mathrm{~s}^{-1}$, which is close to the $V_{\infty}-\lambda_{\infty}$ relation we derived with Approach 2 (blue dashed line in Figure 8). This result seems to indicate that the assumptions made in Approach 1 may be inferior to those made in Approach 2. In other words, the flow peak intensities in the E-step $4 \mathrm{He}$ map likely include a contribution from the secondary He population. The difference in flow speeds and longitudes for the secondary populations obtained from Approach 1 over that from Approach 2 may indicate that there is a trend of the derived values with the E-steps used for the analysis. Therefore, an analysis of the $I B E X$-Lo He observations separately for all E-steps (1 through 4) with a parameter fitting method may be an appropriate extension, which we leave to future studies.

\section{SUMMARY AND CONCLUSIONS}

In this paper, we generated all-sky He maps in the energy range $15-110 \mathrm{eV}$ (E-step 1-4) and $\mathrm{O}$ maps in the energy range 280-600 eV (E-step 5-6) to investigate the directional distribution of primary and secondary ISN $\mathrm{He}$ and $\mathrm{O}$ populations. We applied the sputtering corrections to these maps. In the He maps, the sputtering correction removes contributions due to heliospheric $\mathrm{H}$ ENAs and the ISN $\mathrm{O}$ and $\mathrm{Ne}$ gas flow. However, the contribution from the ISN H gas flow is still present in the He map in E-step 1 between $\lambda=260^{\circ}-300^{\circ}$. The primary ISN He gas is seen between $\lambda=200^{\circ}-260^{\circ}$, and the secondary He population is observed in the longitude range $160^{\circ}-200^{\circ}$ with a broad directional distribution. The secondary $\mathrm{He}$ population is not clearly separated from the primary ISN He flow. On the other hand, the ISN O gas is seen between $\lambda=200^{\circ}-250^{\circ}$ in the E-step 6 $\mathrm{O}$ map and the secondary $\mathrm{O}$ in the longitude range $165^{\circ}-195^{\circ}$ in the E-step $5 \mathrm{O}$ map. In the E-step $5 \mathrm{O}$ map, sputter products of the primary ISN O atoms as obtained from the E-step 6 map that masquerade as converted $\mathrm{O}$ ions are removed by the sputtering correction, and the residual emission should represent the secondary $\mathrm{O}$ population, which has a lower bulk energy than the primary ISN O gas.

We use two approaches to separate the secondary $\mathrm{He}$ population from the primary ISN He gas. In Approach 1, we assume that the count rates in E-step 4 represent the primary ISN He gas. To obtain the count rate of the secondary $\mathrm{He}$ population, we subtract the normalized count rates in E-step 4 from the normalized count rates in E-steps 1-3. Approach 1 suggests an observer location for the maximum secondary $\mathrm{He}$ population of $\lambda_{\mathrm{Obs}, \mathrm{Sec}, \mathrm{He}} \sim 119^{\circ}$ at 1 au in ecliptic longitude. In Approach 2, we assume that the Gaussian distribution with the average peak longitude and its width represents the primary ISN He gas. In this second approach, the observer location for the observation of the maximum secondary He population is $\lambda_{\mathrm{Obs}, \mathrm{Sec}, \mathrm{He}} \sim 98^{\circ}$ in ecliptic longitude.

In the E-step $5 \mathrm{O}$ map, the secondary $\mathrm{O}$ population is clearly separated from the primary ISN O gas even after the sputtering corrections. The observer location of the maximum of the secondary $\mathrm{O}$ population is $\lambda_{\mathrm{Obs}, \mathrm{Sec}, \mathrm{O}} \sim 89^{\circ}$ at 1 au in ecliptic longitude. We explain the difference in the peak location of the two secondary populations by a slower thermal speed and thus effective flow speed of secondary O compared to He due to its larger atomic mass. Using the hyperbolic trajectory equation, we estimate that the flow longitudes of the secondary ISN He and $\mathrm{O}$ populations are in the longitude range $55^{\circ}-88^{\circ}$ and $61^{\circ}$ $77^{\circ}$, respectively, assuming a plasma temperature of $9500-35,000 \mathrm{~K}$. These results are consistent with a possible asymmetry of the outer heliosheath due to the action of the interstellar magnetic field and with a narrower velocity distribution of secondary $\mathrm{O}$ compared to secondary He.

This work was supported by the Interstellar Boundary Explorer mission as a part of the NASA Explorer Program. M. A.K. and M.B. was supported by National Science Centre, Poland, grant 2015-18-M-ST9-00036.

\section{APPENDIX A}

\section{SPUTTERING CORRECTION FOR IBEX-Lo MAPS}

In the following, we derive the sputtering correction for the IBEX-Lo maps. As we discuss in Section 3.2, Equation (1) represents the measured count rate of negative hydrogen ions at E-step $i$ in terms of flux. In Equation (1), the geometric factor $G_{i k}^{\mathrm{XS}}$ characterizes the product of effective instrument area, solid-angle acceptance, energy resolution of ESA, and efficiency. The efficiency depends on the incident species "S," the incident energy (in discrete step $k$ ), the detected ion species "X," and the ESA-step $i$. The efficiency is determined by careful analysis of calibration data. Beams of several neutral species were used to study the response of the IBEX-Lo sensor. 

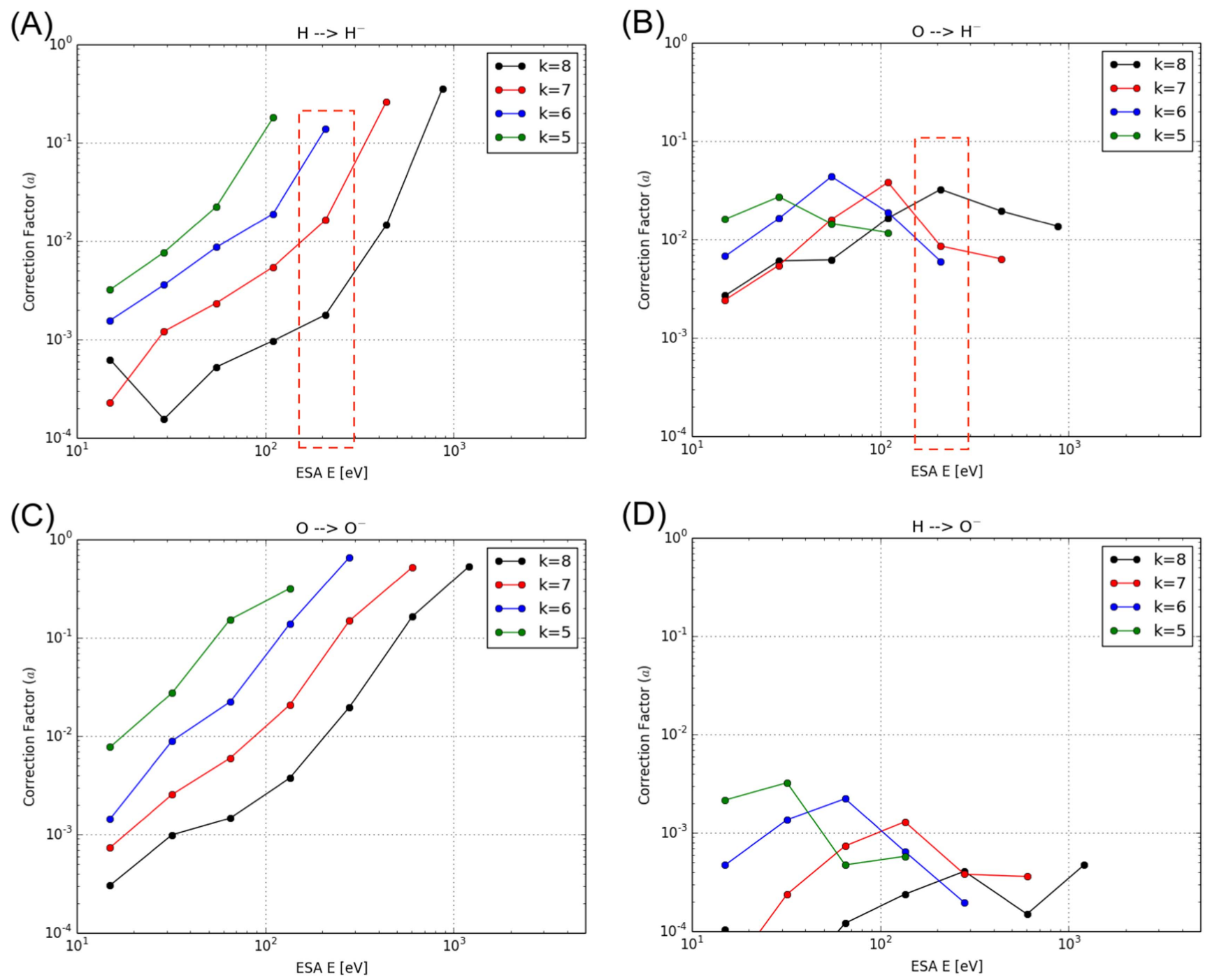

Figure 10. Sputtering-correction factors in the $\mathrm{H}^{-}$count-rate maps due to the incoming $\mathrm{H}$ (panel A) and $\mathrm{O}$ (panel $\mathrm{B}$ ) and in the $\mathrm{O}^{-}$count-rate maps due to the incoming $\mathrm{O}$ (panel $\mathrm{C}$ ) and $\mathrm{H}$ (panel D). " $k$ " is an index of the E-step for the incident energy. The red dashed box represents an example for the $\mathrm{H}^{-}$count-rate map in E-step $5\left(E_{5}=209 \mathrm{eV}\right)$.

Table 4

Values of the Sputtering-correction Factors $\left(\alpha_{i k}^{\mathrm{XS}}\right)$ and Geometric Factors $\left(G_{i k}^{\mathrm{XS}}\right.$, $\mathrm{cm}^{2} \mathrm{sr} \mathrm{keV} / \mathrm{keV}$ ) that we Use in this Study

\begin{tabular}{cccccc}
\hline \hline$k$ & $i$ & $G_{i k}^{\mathrm{H}^{-} \mathrm{H}}$ & $\alpha_{i k}^{\mathrm{H}^{-} \mathrm{H}}$ & $G_{i k}^{\mathrm{O}^{-} \mathrm{O}}$ & $\alpha_{i k}^{\mathrm{O}^{-} \mathrm{O}}$ \\
\hline 8 & 7 & $5.538 \mathrm{E}-05$ & 0.358 & $8.111 \mathrm{E}-05$ & 0.531 \\
& 6 & $4.492 \mathrm{E}-06$ & 0.015 & $5.001 \mathrm{E}-05$ & 0.163 \\
& 5 & $1.152 \mathrm{E}-06$ & 0.002 & $1.292 \mathrm{E}-05$ & 0.020 \\
7 & 6 & $2.718 \mathrm{E}-05$ & 0.262 & $8.326 \mathrm{E}-05$ & 0.518 \\
& 5 & $3.574 \mathrm{E}-06$ & 0.016 & $5.133 \mathrm{E}-05$ & 0.148 \\
6 & 5 & $8.236 \mathrm{E}-06$ & 0.139 & $9.715 \mathrm{E}-05$ & 0.655 \\
\hline
\end{tabular}

The energy of these beams ranged $0.01-2 \mathrm{keV}$. Specific energies have been selected that matched the center energy of the energy steps of the ESA. The ESA allows ions to pass when their individual kinetic energy is within a specific range. For these measurements, we refer to the center energy of the ESA and not to the energy of an individual incoming atom. Through the instrument, we only know the corresponding energy step for the incoming atoms, not their individual energies. Therefore, we use the center energy of the E-step instead of the energy of an incoming atom. In the symbol of the geometric factor, the first letter of the subscript indicates an observed ESA-step and the second letter indicates an energy step associated with the incident energy. The differential fluxes simply represents the observed count rate divided by the observed energy times the geometric factor at the observed energy:

$$
J_{k}^{\mathrm{S}}=\frac{C_{i}^{\mathrm{S}}}{G_{i k}^{\mathrm{XS}} E_{i}}
$$

On the right-hand side of Equation (1), the first term indicates a count rate of converted hydrogen ions and the flux $J_{i}^{\mathrm{H}}$ can be replaced with $C_{i}^{\mathrm{H}} /\left(G_{i i}^{\mathrm{H}^{-} \mathrm{H}} E_{i}\right)$. Since, however, the other three terms represent contributions due to the sputtering products, the fluxes should correspond to the incident neutral atoms with higher energies than the center energy of the observed ESAstep $i$. Thus, the fluxes $J_{k}^{\mathrm{S}}$ is replaced with $C_{k}^{\mathrm{S}} /\left(G_{k k}^{\mathrm{SS}} E_{k}\right)$ for $\mathrm{H}$ and O. For He, the flux $J_{k}^{\mathrm{He}}$ can be written as $C_{k}^{\mathrm{He}} /\left(G_{k k}^{\mathrm{H}-\mathrm{He}} E_{k}\right)$ because helium atoms almost do not produce stable negative helium ions (Wurz et al. 2008). Therefore, we rewrite 
Table 5

Geometric Factors of Triple Coincidence Events for $\mathrm{H} \rightarrow \mathrm{H}^{-}$

\begin{tabular}{lcccccccc}
\hline \hline$i / k$ & 1 & 2 & 3 & 4 & 5 & 6 & 7 \\
\hline 1 & $7.29 \mathrm{E}-06$ & $4.23 \mathrm{E}-06$ & $5.70 \mathrm{E}-06$ & $1.09 \mathrm{E}-06$ & $1.08 \mathrm{E}-06$ & $1.29 \mathrm{E}-06$ & $6.92 \mathrm{E}-07$ \\
2 & $0.00 \mathrm{E}+00$ & $1.41 \mathrm{E}-05$ & $1.78 \mathrm{E}-05$ & $2.30 \mathrm{E}-06$ & $1.33 \mathrm{E}-06$ & $1.53 \mathrm{E}-06$ & $1.90 \mathrm{E}-06$ \\
3 & $0.00 \mathrm{E}+00$ & $0.00 \mathrm{E}+00$ & $2.17 \mathrm{E}-05$ & $9.48 \mathrm{E}-06$ & $2.05 \mathrm{E}-06$ & $1.95 \mathrm{E}-06$ & $1.95 \mathrm{E}-06$ \\
4 & $0.00 \mathrm{E}+00$ & $0.00 \mathrm{E}+00$ & $0.00 \mathrm{E}+00$ & $2.43 \mathrm{E}-05$ & $8.22 \mathrm{E}-06$ & $2.13 \mathrm{E}-06$ & $2.26 \mathrm{E}-06$ \\
5 & $0.00 \mathrm{E}+00$ & $0.00 \mathrm{E}+00$ & $0.00 \mathrm{E}+00$ & $0.00 \mathrm{E}+00$ & $2.41 \mathrm{E}-05$ & $8.24 \mathrm{E}-06$ & $3.57 \mathrm{E}-06$ & $1.19 \mathrm{E}-06$ \\
6 & $0.00 \mathrm{E}+00$ & $0.00 \mathrm{E}+00$ & $0.00 \mathrm{E}+00$ & $0.00 \mathrm{E}+00$ & $0.00 \mathrm{E}+00$ & $2.82 \mathrm{E}-05$ & $2.72 \mathrm{E}-05$ \\
7 & $0.00 \mathrm{E}+00$ & $0.00 \mathrm{E}+00$ & $0.00 \mathrm{E}+00$ & $0.00 \mathrm{E}+00$ & $0.00 \mathrm{E}+00$ & $0.00 \mathrm{E}+00$ & $5.23 \mathrm{E}-05$ \\
8 & $0.00 \mathrm{E}+00$ & $0.00 \mathrm{E}+00$ & $0.00 \mathrm{E}+00$ & $0.00 \mathrm{E}+00$ & $0.00 \mathrm{E}+00$ & $0.00 \mathrm{E}+00$ & $0.00 \mathrm{E}+00$ & $7.54 \mathrm{E}-05$ \\
& & &
\end{tabular}

Note. $i$ is an ESA E-step and $k$ is an incident energy step.

Table 6

Geometric Factors of Triple Coincidence Events for $\mathrm{H} \rightarrow \mathrm{O}^{-}$

\begin{tabular}{lcccccccc}
\hline \hline$i / k$ & 1 & 2 & 3 & 4 & 5 & 6 & 7 \\
\hline 1 & $2.09 \mathrm{E}-08$ & $3.05 \mathrm{E}-08$ & $4.46 \mathrm{E}-08$ & $1.72 \mathrm{E}-07$ & $7.24 \mathrm{E}-07$ & $3.88 \mathrm{E}-07$ & $9.89 \mathrm{E}-08$ \\
2 & $0.00 \mathrm{E}+00$ & $5.13 \mathrm{E}-08$ & $4.03 \mathrm{E}-08$ & $1.93 \mathrm{E}-08$ & $5.08 \mathrm{E}-07$ & $5.24 \mathrm{E}-07$ & $3.38 \mathrm{E}-07$ \\
3 & $0.00 \mathrm{E}+00$ & $0.00 \mathrm{E}+00$ & $3.92 \mathrm{E}-08$ & $3.97 \mathrm{E}-08$ & $3.65 \mathrm{E}-08$ & $4.23 \mathrm{E}-07$ & $5.15 \mathrm{E}-07$ \\
4 & $0.00 \mathrm{E}+00$ & $0.00 \mathrm{E}+00$ & $0.00 \mathrm{E}+00$ & $9.44 \mathrm{E}-08$ & $2.15 \mathrm{E}-08$ & $5.90 \mathrm{E}-08$ & $4.36 \mathrm{E}-07$ \\
5 & $0.00 \mathrm{E}+00$ & $0.00 \mathrm{E}+00$ & $0.00 \mathrm{E}+00$ & $0.00 \mathrm{E}+00$ & $6.05 \mathrm{E}-08$ & $8.67 \mathrm{E}-09$ & $6.20 \mathrm{E}-08$ & $2.30 \mathrm{E}-07$ \\
6 & $0.00 \mathrm{E}+00$ & $0.00 \mathrm{E}+00$ & $0.00 \mathrm{E}+00$ & $0.00 \mathrm{E}+00$ & $0.00 \mathrm{E}+00$ & $1.55 \mathrm{E}-08$ & $2.72 \mathrm{E}-08$ \\
7 & $0.00 \mathrm{E}+00$ & $0.00 \mathrm{E}+00$ & $0.00 \mathrm{E}+00$ & $0.00 \mathrm{E}+00$ & $0.00 \mathrm{E}+00$ & $0.00 \mathrm{E}+00$ & $0.00 \mathrm{E}+00$ & $3.35 \mathrm{E}-08$ \\
8 & $0.00 \mathrm{E}+00$ & $0.00 \mathrm{E}+00$ & $0.00 \mathrm{E}+00$ & $0.00 \mathrm{E}+00$ & $0.00 \mathrm{E}+00$ & $0.00 \mathrm{E}+00$ & $0.00 \mathrm{E}+00$ & $0.00 \mathrm{E}+00$ \\
\hline
\end{tabular}

Table 7

Geometric Factors of Triple Coincidence Events for $\mathrm{O} \rightarrow \mathrm{O}^{-}$

\begin{tabular}{lccccccc}
\hline \hline$i / k$ & 1 & 2 & 3 & 4 & 5 & 6 & 7 \\
\hline 1 & $3.55 \mathrm{E}-05$ & $2.31 \mathrm{E}-05$ & $3.48 \mathrm{E}-05$ & $3.46 \mathrm{E}-05$ & $1.04 \mathrm{E}-05$ & $3.97 \mathrm{E}-06$ & $4.72 \mathrm{E}-06$ \\
2 & $0.00 \mathrm{E}+00$ & $3.98 \mathrm{E}-05$ & $5.25 \mathrm{E}-05$ & $9.10 \mathrm{E}-05$ & $1.73 \mathrm{E}-05$ & $1.15 \mathrm{E}-05$ & $7.68 \mathrm{E}-06$ \\
3 & $0.00 \mathrm{E}+00$ & $0.00 \mathrm{E}+00$ & $6.36 \mathrm{E}-05$ & $2.10 \mathrm{E}-04$ & $4.76 \mathrm{E}-05$ & $1.42 \mathrm{E}-05$ & $8.86 \mathrm{E}-06$ \\
4 & $0.00 \mathrm{E}+00$ & $0.00 \mathrm{E}+00$ & $0.00 \mathrm{E}+00$ & $7.29 \mathrm{E}-05$ & $4.74 \mathrm{E}-05$ & $4.25 \mathrm{E}-05$ & $1.48 \mathrm{E}-06$ \\
5 & $0.00 \mathrm{E}+00$ & $0.00 \mathrm{E}+00$ & $0.00 \mathrm{E}+00$ & $0.00 \mathrm{E}+00$ & $7.24 \mathrm{E}-05$ & $9.71 \mathrm{E}-05$ & $5.13 \mathrm{E}-05$ \\
6 & $0.00 \mathrm{E}+00$ & $0.00 \mathrm{E}+00$ & $0.00 \mathrm{E}+00$ & $0.00 \mathrm{E}+00$ & $0.00 \mathrm{E}+00$ & $6.89 \mathrm{E}-05$ & $8.33 \mathrm{E}-05$ \\
7 & $0.00 \mathrm{E}+00$ & $0.00 \mathrm{E}+00$ & $0.00 \mathrm{E}+00$ & $0.00 \mathrm{E}+00$ & $0.00 \mathrm{E}+00$ & $0.00 \mathrm{E}+00$ & $8.01 \mathrm{E}-06$ \\
8 & $0.00 \mathrm{E}+00$ & $0.00 \mathrm{E}+00$ & $0.00 \mathrm{E}+00$ & $0.00 \mathrm{E}+00$ & $0.00 \mathrm{E}+00$ & $0.00 \mathrm{E}+00$ & $0.00 \mathrm{E}+00$ \\
\hline
\end{tabular}

Table 8

Geometric Factors of Triple Coincidence Events for $\mathrm{O} \rightarrow \mathrm{H}^{-}$

\begin{tabular}{lccccccc}
\hline \hline$i / k$ & 1 & 2 & 3 & 4 & 5 & 6 & 7 \\
\hline 1 & $2.15 \mathrm{E}-06$ & $3.01 \mathrm{E}-06$ & $1.50 \mathrm{E}-05$ & $3.01 \mathrm{E}-05$ & $2.16 \mathrm{E}-05$ & $1.86 \mathrm{E}-05$ & $1.57 \mathrm{E}-05$ \\
2 & $0.00 \mathrm{E}+00$ & $2.34 \mathrm{E}-07$ & $5.96 \mathrm{E}-07$ & $9.84 \mathrm{E}-06$ & $1.89 \mathrm{E}-05$ & $2.34 \mathrm{E}-05$ & $1.82 \mathrm{E}-05$ \\
3 & $0.00 \mathrm{E}+00$ & $0.00 \mathrm{E}+00$ & $1.66 \mathrm{E}-07$ & $1.29 \mathrm{E}-06$ & $5.31 \mathrm{E}-06$ & $3.28 \mathrm{E}-05$ & $2.80 \mathrm{E}-05$ \\
4 & $0.00 \mathrm{E}+00$ & $0.00 \mathrm{E}+00$ & $0.00 \mathrm{E}+00$ & $3.96 \mathrm{E}-07$ & $2.16 \mathrm{E}-06$ & $7.10 \mathrm{E}-06$ & $3.34 \mathrm{E}-05$ \\
5 & $0.00 \mathrm{E}+00$ & $0.00 \mathrm{E}+00$ & $0.00 \mathrm{E}+00$ & $0.00 \mathrm{E}+00$ & $4.55 \mathrm{E}-07$ & $1.19 \mathrm{E}-06$ & $3.96 \mathrm{E}-06$ \\
6 & $0.00 \mathrm{E}+00$ & $0.00 \mathrm{E}+00$ & $0.00 \mathrm{E}+00$ & $0.00 \mathrm{E}+00$ & $0.00 \mathrm{E}+00$ & $1.00 \mathrm{E}-06$ & $1.39 \mathrm{E}-06$ \\
7 & $0.00 \mathrm{E}+00$ & $0.00 \mathrm{E}+00$ & $0.00 \mathrm{E}+00$ & $0.00 \mathrm{E}+00$ & $0.00 \mathrm{E}+00$ & $0.00 \mathrm{E}+00$ & $4.71 \mathrm{E}-07$ \\
8 & $0.00 \mathrm{E}+00$ & $0.00 \mathrm{E}+00$ & $0.00 \mathrm{E}+00$ & $0.00 \mathrm{E}+00$ & $0.00 \mathrm{E}+00$ & $0.00 \mathrm{E}+00$ & $0.00 \mathrm{E}+00$ \\
\hline
\end{tabular}

Equation (1) in terms of the count rate:

$$
\begin{aligned}
C_{i}^{\mathrm{H}^{-}}= & C_{i}^{\mathrm{H}}+\sum_{k>i} \frac{G_{i k}^{\mathrm{H}^{-} \mathrm{H}} E_{i}}{G_{k k}^{\mathrm{H}^{-} \mathrm{H}} E_{k}} C_{k}^{\mathrm{H}}+\sum_{k \geqslant i} \frac{G_{i k}^{\mathrm{H}^{-} \mathrm{He}} E_{i}}{G_{k k}^{\mathrm{H}^{-} \mathrm{He}} E_{k}} C_{k}^{\mathrm{He}} \\
& +\sum_{k \geqslant i} \frac{G_{i k}^{\mathrm{H}^{-} \mathrm{O}} E_{i}}{G_{k k}^{\mathrm{O}^{-} \mathrm{O}} E_{k}} C_{k}^{\mathrm{O}}
\end{aligned}
$$

Here we define a sputtering-correction factor $\alpha_{i k}^{\mathrm{XS}}$ :

$$
\alpha_{i k}^{\mathrm{XS}}=\frac{G_{i k}^{\mathrm{XS}} E_{i}}{G_{k k}^{\mathrm{SS}} E_{k}}
$$

Extensive testing during the pre-flight instrument calibration with pure neutral hydrogen, helium, and oxygen beams derived the geometric factors. The top two panels in Figure 10 shows the sputtering-correction factors in the $\mathrm{H}^{-}$count-rate maps due 
Table 9

Geometric Factors of Triple Coincidence Events for $\mathrm{He} \rightarrow \mathrm{H}^{-}$

\begin{tabular}{lcccccccc}
\hline \hline$i / k$ & 1 & 2 & 3 & 4 & 5 & 6 & 7 \\
\hline 1 & $8.23 \mathrm{E}-06$ & $2.24 \mathrm{E}-05$ & $3.63 \mathrm{E}-05$ & $4.89 \mathrm{E}-05$ & $2.57 \mathrm{E}-05$ & $7.70 \mathrm{E}-06$ & $7.43 \mathrm{E}-06$ \\
2 & $0.00 \mathrm{E}+00$ & $7.79 \mathrm{E}-06$ & $3.81 \mathrm{E}-05$ & $6.03 \mathrm{E}-05$ & $2.60 \mathrm{E}-05$ & $7.62 \mathrm{E}-06$ & $6.19 \mathrm{E}-06$ \\
3 & $0.00 \mathrm{E}+00$ & $0.00 \mathrm{E}+00$ & $1.06 \mathrm{E}-05$ & $5.22 \mathrm{E}-05$ & $2.76 \mathrm{E}-05$ & $7.71 \mathrm{E}-06$ & $5.49 \mathrm{E}-06$ \\
4 & $0.00 \mathrm{E}+00$ & $0.00 \mathrm{E}+00$ & $0.00 \mathrm{E}+00$ & $4.89 \mathrm{E}-06$ & $1.92 \mathrm{E}-05$ & $1.19 \mathrm{E}-06$ & $4.59 \mathrm{E}-06$ \\
5 & $0.00 \mathrm{E}+00$ & $0.00 \mathrm{E}+00$ & $0.00 \mathrm{E}+00$ & $0.00 \mathrm{E}+00$ & $7.00 \mathrm{E}-07$ & $3.79 \mathrm{E}-07$ & $1.17 \mathrm{E}-06$ \\
6 & $0.00 \mathrm{E}+00$ & $0.00 \mathrm{E}+00$ & $0.00 \mathrm{E}+00$ & $0.00 \mathrm{E}+00$ & $0.00 \mathrm{E}+00$ & $3.05 \mathrm{E}-07$ & $1.50 \mathrm{E}-06$ \\
7 & $0.00 \mathrm{E}+00$ & $0.00 \mathrm{E}+00$ & $0.00 \mathrm{E}+00$ & $0.00 \mathrm{E}+00$ & $0.00 \mathrm{E}+00$ & $0.00 \mathrm{E}+00$ & $1.88 \mathrm{E}-00$ \\
8 & $0.00 \mathrm{E}+00$ & $0.00 \mathrm{E}+00$ & $0.00 \mathrm{E}+00$ & $0.00 \mathrm{E}+00$ & $0.00 \mathrm{E}+00$ & $0.00 \mathrm{E}+00$ & $0.00 \mathrm{E}+00$ & $2.67 \mathrm{E}-06$ \\
& & &
\end{tabular}

Table 10

Geometric Factors of Triple Coincidence Events for $\mathrm{He} \rightarrow \mathrm{O}^{-}$

\begin{tabular}{lcccccccc}
\hline \hline$i / k$ & 1 & 2 & 3 & 4 & 5 & 6 & 7 \\
\hline 1 & $2.49 \mathrm{E}-08$ & $1.48 \mathrm{E}-07$ & $7.21 \mathrm{E}-08$ & $1.51 \mathrm{E}-06$ & $9.77 \mathrm{E}-07$ & $3.53 \mathrm{E}-07$ & $5.17 \mathrm{E}-07$ \\
2 & $0.00 \mathrm{E}+00$ & $2.36 \mathrm{E}-08$ & $7.85 \mathrm{E}-07$ & $2.04 \mathrm{E}-06$ & $1.41 \mathrm{E}-06$ & $4.79 \mathrm{E}-07$ & $5.33 \mathrm{E}-07$ \\
3 & $0.00 \mathrm{E}+00$ & $0.00 \mathrm{E}+00$ & $1.31 \mathrm{E}-07$ & $1.88 \mathrm{E}-06$ & $2.14 \mathrm{E}-06$ & $6.51 \mathrm{E}-07$ & $6.78 \mathrm{E}-07$ \\
4 & $0.00 \mathrm{E}+00$ & $0.00 \mathrm{E}+00$ & $0.00 \mathrm{E}+00$ & $1.38 \mathrm{E}-07$ & $1.29 \mathrm{E}-06$ & $1.59 \mathrm{E}-07$ & $7.22 \mathrm{E}-07$ \\
5 & $0.00 \mathrm{E}+00$ & $0.00 \mathrm{E}+00$ & $0.00 \mathrm{E}+00$ & $0.00 \mathrm{E}+00$ & $2.59 \mathrm{E}-08$ & $4.56 \mathrm{E}-08$ & $2.05 \mathrm{E}-07$ & $1.21 \mathrm{E}-06$ \\
6 & $0.00 \mathrm{E}+00$ & $0.00 \mathrm{E}+00$ & $0.00 \mathrm{E}+00$ & $0.00 \mathrm{E}+00$ & $0.00 \mathrm{E}+00$ & $2.02 \mathrm{E}-08$ & $1.93 \mathrm{E}-07$ & $4.10 \mathrm{E}-07$ \\
7 & $0.00 \mathrm{E}+00$ & $0.00 \mathrm{E}+00$ & $0.00 \mathrm{E}+00$ & $0.00 \mathrm{E}+00$ & $0.00 \mathrm{E}+00$ & $0.00 \mathrm{E}+00$ & $1.44 \mathrm{E}-08$ & $5.27 \mathrm{E}-07$ \\
8 & $0.00 \mathrm{E}+00$ & $0.00 \mathrm{E}+00$ & $0.00 \mathrm{E}+00$ & $0.00 \mathrm{E}+00$ & $0.00 \mathrm{E}+00$ & $0.00 \mathrm{E}+00$ & $0.00 \mathrm{E}+00$ & $1.56 \mathrm{E}-08$ \\
\hline
\end{tabular}

Table 11

Geometric Factors of Triple Coincidence Events for $\mathrm{Ne} \rightarrow \mathrm{H}^{-}$

\begin{tabular}{|c|c|c|c|c|c|c|c|c|}
\hline$i / k$ & 1 & 2 & 3 & 4 & 5 & 6 & 7 & 8 \\
\hline 1 & $0.00 \mathrm{E}+00$ & 4.07E-06 & $0.00 \mathrm{E}+00$ & $1.52 \mathrm{E}-04$ & $0.00 \mathrm{E}+00$ & $0.00 \mathrm{E}+00$ & $0.00 \mathrm{E}+00$ & $3.12 \mathrm{E}-05$ \\
\hline 2 & $0.00 \mathrm{E}+00$ & $3.22 \mathrm{E}-07$ & $0.00 \mathrm{E}+00$ & $6.20 \mathrm{E}-05$ & $1.72 \mathrm{E}-04$ & $1.46 \mathrm{E}-04$ & $2.92 \mathrm{E}-05$ & 4.03E-05 \\
\hline 3 & $0.00 \mathrm{E}+00$ & $0.00 \mathrm{E}+00$ & 2.04E-07 & 2.38E-06 & 4.80E-05 & $1.85 \mathrm{E}-04$ & $9.53 \mathrm{E}-05$ & 4.72E-05 \\
\hline 4 & $0.00 \mathrm{E}+00$ & $0.00 \mathrm{E}+00$ & $0.00 \mathrm{E}+00$ & $2.57 \mathrm{E}-08$ & $2.59 \mathrm{E}-06$ & $2.48 \mathrm{E}-05$ & $1.10 \mathrm{E}-04$ & $5.42 \mathrm{E}-05$ \\
\hline 5 & $0.00 \mathrm{E}+00$ & $0.00 \mathrm{E}+00$ & $0.00 \mathrm{E}+00$ & $0.00 \mathrm{E}+00$ & 6.61E-08 & $5.04 \mathrm{E}-07$ & $1.16 \mathrm{E}-05$ & $5.23 \mathrm{E}-05$ \\
\hline 6 & $0.00 \mathrm{E}+00$ & $0.00 \mathrm{E}+00$ & $0.00 \mathrm{E}+00$ & $0.00 \mathrm{E}+00$ & $0.00 \mathrm{E}+00$ & $1.21 \mathrm{E}-07$ & 5.12E-07 & 2.65E-06 \\
\hline 7 & $0.00 \mathrm{E}+00$ & $0.00 \mathrm{E}+00$ & $0.00 \mathrm{E}+00$ & $0.00 \mathrm{E}+00$ & $0.00 \mathrm{E}+00$ & $0.00 \mathrm{E}+00$ & $9.15 \mathrm{E}-08$ & 4.72E-07 \\
\hline 8 & $0.00 \mathrm{E}+00$ & $0.00 \mathrm{E}+00$ & $0.00 \mathrm{E}+00$ & $0.00 \mathrm{E}+00$ & $0.00 \mathrm{E}+00$ & $0.00 \mathrm{E}+00$ & $0.00 \mathrm{E}+00$ & 4.85E-08 \\
\hline
\end{tabular}

Table 12

Geometric Factors of Triple Coincidence Events for $\mathrm{Ne} \rightarrow \mathrm{O}^{-}$

\begin{tabular}{lcccccccc}
\hline \hline$i / k$ & 1 & 2 & 3 & 4 & 5 & 6 & 7 \\
\hline 1 & $0.00 \mathrm{E}+00$ & $1.72 \mathrm{E}-06$ & $0.00 \mathrm{E}+00$ & $7.18 \mathrm{E}-06$ & $0.00 \mathrm{E}+00$ & $0.00 \mathrm{E}+00$ & $0.00 \mathrm{E}+00$ & $9.53 \mathrm{E}-06$ \\
2 & $0.00 \mathrm{E}+00$ & $1.72 \mathrm{E}-06$ & $0.00 \mathrm{E}+00$ & $6.50 \mathrm{E}-06$ & $1.83 \mathrm{E}-05$ & $1.87 \mathrm{E}-05$ & $8.52 \mathrm{E}-06$ \\
3 & $0.00 \mathrm{E}+00$ & $0.00 \mathrm{E}+00$ & $2.15 \mathrm{E}-06$ & $1.32 \mathrm{E}-05$ & $2.13 \mathrm{E}-05$ & $3.33 \mathrm{E}-05$ & $2.13 \mathrm{E}-05$ \\
4 & $0.00 \mathrm{E}+00$ & $0.00 \mathrm{E}+00$ & $0.00 \mathrm{E}+00$ & $2.61 \mathrm{E}-06$ & $2.80 \mathrm{E}-05$ & $4.07 \mathrm{E}-05$ & $3.46 \mathrm{E}-05$ \\
5 & $0.00 \mathrm{E}+00$ & $0.00 \mathrm{E}+00$ & $0.00 \mathrm{E}+00$ & $0.00 \mathrm{E}+00$ & $5.25 \mathrm{E}-06$ & $4.39 \mathrm{E}-05$ & $3.59 \mathrm{E}-05$ & $1.92 \mathrm{E}-05$ \\
6 & $0.00 \mathrm{E}+00$ & $0.00 \mathrm{E}+00$ & $0.00 \mathrm{E}+00$ & $0.00 \mathrm{E}+00$ & $0.00 \mathrm{E}+00$ & $7.89 \mathrm{E}-06$ & $3.56 \mathrm{E}-05$ & $2.90 \mathrm{E}-05$ \\
7 & $0.00 \mathrm{E}+00$ & $0.00 \mathrm{E}+00$ & $0.00 \mathrm{E}+00$ & $0.00 \mathrm{E}+00$ & $0.00 \mathrm{E}+00$ & $0.00 \mathrm{E}+00$ & $9.20 \mathrm{E}-06$ & $2.72 \mathrm{E}-05$ \\
8 & $0.00 \mathrm{E}+00$ & $0.00 \mathrm{E}+00$ & $0.00 \mathrm{E}+00$ & $0.00 \mathrm{E}+00$ & $0.00 \mathrm{E}+00$ & $0.00 \mathrm{E}+00$ & $0.00 \mathrm{E}+00$ & $1.05 \mathrm{E}-05$ \\
\hline
\end{tabular}

to the incoming $\mathrm{H}$ and $\mathrm{O}$ atoms, respectively. For instance, the $\mathrm{H}^{-}$count rate in E-step $5\left(C_{5}^{\mathrm{H}^{-}}, E_{5}=209 \mathrm{eV}\right)$ is

$$
C_{5}^{\mathrm{H}^{-}}=C_{5}^{\mathrm{H}}+\sum_{k=6}^{8}\left(\alpha_{5 k}^{\mathrm{H}^{-} \mathrm{H}} C_{k}^{\mathrm{H}}\right)+\sum_{k=5}^{8}\left(\alpha_{5 k}^{\mathrm{H}^{-} \mathrm{O}} C_{k}^{\mathrm{O}}\right)
$$

where we ignore the sputtering term due to $\mathrm{He}$ atoms because we only expect to observe ISN He atoms with the bulk energy of $\sim 131 \mathrm{eV}$ in the spacecraft frame during the winter-spring season of the ISN He gas. The correction factors for the second and third terms in Equation (20) are denoted by the red box in
Figure 10. The bottom two panels in Figure 10 show the sputtering-correction factors in the $\mathrm{O}^{-}$count-rate maps due to the incoming $\mathrm{O}$ and $\mathrm{H}$ atoms, respectively. Table 4 shows the actual values of the sputtering-correction factors and the geometric factors that we use in this study.

\section{APPENDIX B}

\section{GEOMETRIC FACTORS FOR THE IBEX-Lo SENSOR}

In this section, we provide geometric factors of $I B E X$-Lo for four neutral species $(\mathrm{H}, \mathrm{He}, \mathrm{O}$, and $\mathrm{Ne})$. As mentioned in 
Appendix A, the geometric factor in Tables 5-12 is the product of the absolute geometric factor, the energy resolution of the ESA, TOF efficiency, the combined grid transparency, the collection efficiency, the combined conversion and reflection efficiency, and the relative probability of detecting an ion of species "X." The efficiencies and transparencies are explained in detail in the IBEX science operation paper (Schwadron et al. 2009). Those values are based on various calibration runs with neutral beams of four species during final calibration before flight. In Tables 5-12, the indices $i$ and $k$ indicate the ESA and incident energy steps, respectively, as described in Section 3.2 and Appendix A.

\section{REFERENCES}

Bochsler, P., Petersen, L., Möbius, E., et al. 2012, ApJS, 198, 13 Bzowski, M., Kubiak, M. A., Hłond, M., et al. 2014, A\&A, 569, A8 Bzowski, M., Kubiak, M. A., Möbius, E., et al. 2012, ApJS, 198, 12 Bzowski, M., Swaczyna, P., Kubiak, M., et al. 2015, ApJS, 220, 28

Chalov, S. V., Alexashov, D. B., McComas, D. J., et al. 2010, ApJL, 716, L99 Fuselier, S. A., Allegrini, F., Bzowski, M., et al. 2012, ApJ, 754, 14 Fuselier, S. A., Allegrini, F., Bzowski, M., et al. 2014, ApJ, 784, 89 Fuselier, S. A., Bochsler, P., Chornay, D., et al. 2009, SSRv, 146, 117 Galli, A., Wurz, P., Fuselier, S. A., et al. 2014, ApJ, 796, 9

Galli, A., Wurz, P., Park, J., et al. 2015, ApJS, 220, 30

Galli, A., Wurz, P., Schwadron, N. A., et al. 2016, ApJ, 821, 107

Gruntman, M., Roelof, E. C., Mitchell, D. G., et al. 2001, JGR, 106, 15767

Grzedzielski, S., Swaczyna, P., \& Bzowski, M. , 2013, A\&A, 549, A76

Heerikhuisen, J., Pogorelov, N. V., Zank, G. P., et al. 2010, ApJL, 708, L126 Izmodenov, V. V., Lallement, R., \& Geiss, J. 1999, A\&A, 344, 317

Izmodenov, V. V., Malama, Y. G., Gloeckler, G., \& Geiss, J. 2004, A\&A, 414, L29

Izmodenov, V. V., Malama, Y. G., \& Lallement, R. 1997, A\&A, 317, 193
Izmodenov, V. V., Malama, Y. G., \& Ruderman, M. S. 2005, A\&A, 429, 1069 Izmodenov, V. V., Malama, Y. G., Ruderman, M. S., et al. 2009, SSRv, 146,329

Katushkina, O. A., Izmodenov, V. V., Wood, B. E., et al. 2014, ApJ, 789, 80

Kubiak, M. A., Bzowski, M., Sokół, J. M., et al. 2014, ApJS, 213, 29

Kubiak, M. A., Swaczyna, P., Bzowski, M., et al. 2016, ApJS, 223, 35

Lee, M., Möbius, E., \& Leonard, T. W. 2015, ApJS, 220, 23

Lee, M. A., Kucharek, H., Möbius, E., et al. 2012, ApJS, 198, 10

Leonard, T. W., Möbius, E., Bzowski, M., et al. 2015, ApJ, 804, 42

Malama, Y. G., Izmodenov, V. V., \& Chalov, S. V. 2006, A\&A, 445, 693

McComas, D. J. \& IBEX Science Team 2012, in AIP Conf. Proc. 1500 (Palm Springs, CA: AIP), 222

McComas, D. J., Allegrini, F., Bochsler, P., et al. 2009a, SSRv, 146, 11

McComas, D. J., Allegrini, F., Bochsler, P., et al. 2009b, Sci, 326, 959

McComas, D. J., Allegrini, F., Bzowski, M., et al. 2014, ApJS, 213, 20

McComas, D. J., Bzowski, M., Frisch, P., et al. 2010, JGR, 115, A09113

McComas, D. J., Bzowski, M., Frisch, P. C., et al. 2015a, ApJ, 801, 28

McComas, D. J., Bzowski, M., Fuselier, S. A., et al. 2015b, ApJS, 220, 22

McComas, D. J., Carrico, J. P., Hautamaki, B., et al. 2011, SpWea, 9, S11002

Möbius, E., Bochsler, P., Bzowski, M., et al. 2009, Sci, 326, 969

Möbius, E., Bochsler, P., Bzowski, M., et al. 2012, ApJS, 198, 11

Möbius, E., Bzowski, M., Frisch, P. C., et al. 2015a, ApJS, 220, 24

Möbius, E., Bzowski, M., Fuselier, S. A., et al. 2015b, JPhCS, 577, 012019

Park, J., Kucharek, H., Möbius, E., et al. 2014, ApJ, 795, 97

Park, J., Kucharek, H., Möbius, E., et al. 2015, ApJS, 220, 34

Saul, L., Wurz, P., Rodriguez, D., et al. 2012, ApJS, 198, 14

Schwadron, N. A., Crew, G., Vanderspek, R., et al. 2009, SSRv, 146, 207

Schwadron, N. A., Möbius, E., Fuselier, S. A., et al. 2014, ApJS, 215, 13

Schwadron, N. A., Möbius, E., Kucharek, H., et al. 2013, ApJ, 775, 86

Slavin, J. D., \& Frisch, P. C. 2008, A\&A, 491, 53

Sokół, J. M., Bzowski, M., Kubiak, M. A., et al. 2015, ApJS, 220, 27

Swaczyna, P., Bzowski, M., Kubiak, M. A., et al. 2015, ApJS, 220, 26

Wood, B. E., Müller, H. R., \& Witte, M. 2015, ApJ, 801, 62

Wurz, P., Saul, L., Scheer, J. A., et al. 2008, JAP, 103, 054904

Zieger, B., Opher, M., Schwadron, N. A., et al. 2013, GRL, 40, 2923 